\title{
AIP
}

\section{Direct simulation of proton-coupled electron transfer across multiple}

regimes

Joshua S. Kretchmer and Thomas F. Miller

Citation: J. Chem. Phys. 138, 134109 (2013); doi: 10.1063/1.4797462

View online: http://dx.doi.org/10.1063/1.4797462

View Table of Contents: http://jcp.aip.org/resource/1/JCPSA6/v138/i13

Published by the American Institute of Physics.

\section{Additional information on J. Chem. Phys.}

Journal Homepage: http://jcp.aip.org/

Journal Information: http://jcp.aip.org/about/about_the_journal

Top downloads: http://jcp.aip.org/features/most_downloaded

Information for Authors: http://jcp.aip.org/authors

\section{ADVERTISEMENT}

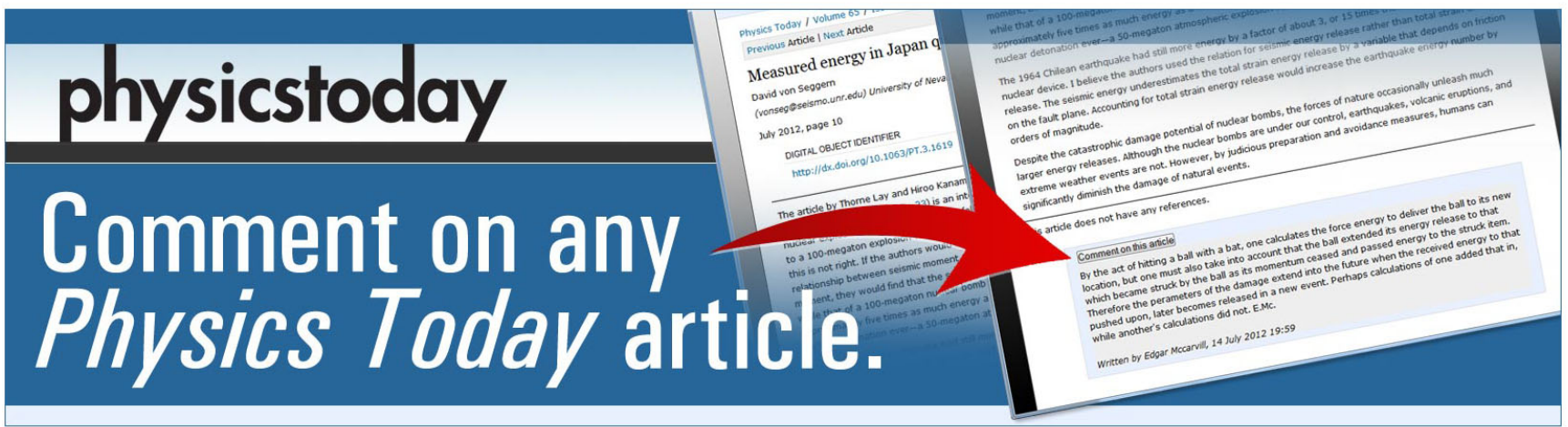




\title{
Direct simulation of proton-coupled electron transfer across multiple regimes
}

\author{
Joshua S. Kretchmer and Thomas F. Miller III \\ Division of Chemistry and Chemical Engineering, California Institute of Technology, Pasadena, \\ California 91125, USA
}

(Received 18 January 2013; accepted 10 March 2013; published online 2 April 2013)

\begin{abstract}
The coupled transfer of electrons and protons is a central feature of biological and molecular catalysis, yet fundamental aspects of these reactions remain poorly understood. In this study, we extend the ring polymer molecular dynamics (RPMD) method to enable direct simulation of proton-coupled electron transfer (PCET) reactions across a wide range of physically relevant regimes. In a systembath model for symmetric, co-linear PCET in the condensed phase, RPMD trajectories reveal distinct kinetic pathways associated with sequential and concerted PCET reaction mechanisms, and it is demonstrated that concerted PCET proceeds by a solvent-gating mechanism in which the reorganization energy is mitigated by charge cancellation among the transferring particles. We further employ RPMD to study the kinetics and mechanistic features of concerted PCET reactions across multiple coupling regimes, including the fully non-adiabatic (both electronically and vibrationally nonadiabatic), partially adiabatic (electronically adiabatic, but vibrationally non-adiabatic), and fully adiabatic (both electronically and vibrationally adiabatic) limits. Comparison of RPMD with the results of PCET rate theories demonstrates the applicability of the direct simulation method over a broad range of conditions; it is particularly notable that RPMD accurately predicts the crossover in the thermal reaction rates between different coupling regimes while avoiding a priori assumptions about the PCET reaction mechanism. Finally, by utilizing the connections between RPMD rate theory and semiclassical instanton theory, we show that analysis of ring-polymer configurations in the RPMD transition path ensemble enables the a posteriori determination of the coupling regime for the PCET reaction. This analysis reveals an intriguing and distinct "transient-proton-bridge" mechanism for concerted PCET that emerges in the transition between the proton-mediated electron superexchange mechanism for fully non-adiabatic PCET and the hydrogen atom transfer mechanism for partially adiabatic PCET. Taken together, these results provide a unifying picture of the mechanisms and physical driving forces that govern PCET across a wide range of physical regimes, and they raise the possibility for PCET mechanisms that have not been previously reported. ( 2013 American Institute of Physics. [http://dx.doi.org/10.1063/1.4797462]
\end{abstract}

\section{INTRODUCTION}

Proton-coupled electron transfer (PCET) reactions, in which both an electron and an associated proton undergo reactive transfer (Fig. 1(a)), play an important role in many chemical and biological processes. ${ }^{1-4}$ Key examples include the tyrosine oxidation step of photosystem $\mathrm{II}^{5,6}$ and the protonpumping mechanism of cytochrome $c$ oxidase. ${ }^{7,8}$ Depending on the chronology of the electron- and proton-transfer events and the magnitudes of the electronic and vibrational coupling, a variety of reactive processes can fall under the umbrella of PCET; ${ }^{9-13}$ investigation of the dynamics that govern this full range of behavior provides significant experimental and theoretical challenges, and the characterization of transitions between different regimes of PCET remains incomplete. In this study, we extend the ring polymer molecular dynamics (RPMD) method to allow for the direct simulation of PCET reaction dynamics and to characterize condensedphase PCET reaction mechanisms and thermal rates across a wide range of physically relevant regimes.

\footnotetext{
${ }^{a)}$ Electronic mail: tfm@caltech.edu
}

PCET reactions are typically described (Fig. 1(b)) in terms of the following reactant, intermediate, and product species: ${ }^{1,9,14-16}$

$$
\begin{array}{rc}
\mathrm{D}-\mathrm{H}+\mathrm{A} & (\mathrm{OU}), \\
\mathrm{D}^{-}+[\mathrm{H}-\mathrm{A}]^{+} & (\mathrm{OP}), \\
{[\mathrm{D}-\mathrm{H}]^{+}+\mathrm{A}^{-}} & (\mathrm{RU}), \\
\mathrm{D}+\mathrm{H}-\mathrm{A} & (\mathrm{RP}) .
\end{array}
$$

Here, D and A indicate the donor and acceptor molecules, respectively, and the labels $\mathrm{O} / \mathrm{R}$ and $\mathrm{U} / \mathrm{P}$ indicate the oxidation state (oxidized or reduced) and the protonation state (unprotonated or protonated) of the acceptor molecule. The reactions can be categorized among two groups, sequential and concerted PCET, depending on whether both the electron and proton transfer in a single chemical step (Fig. 1(b)). ${ }^{9}{ }^{14-16}$ Sequential PCET exhibits distinct electron-transfer (ET) and proton-transfer (PT) reaction events separated by a metastable intermediate species; concerted PCET exhibits the transfer of both particles in a single reactive step, bypassing the formation of the OP and RU species in Fig. 1(b). Within these two 
(a)

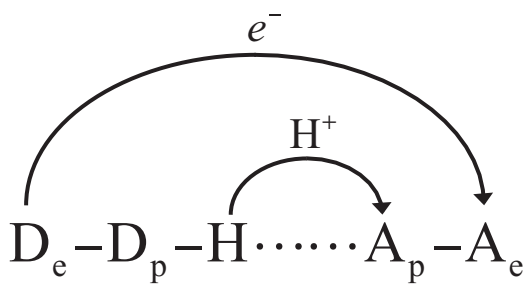

(b)

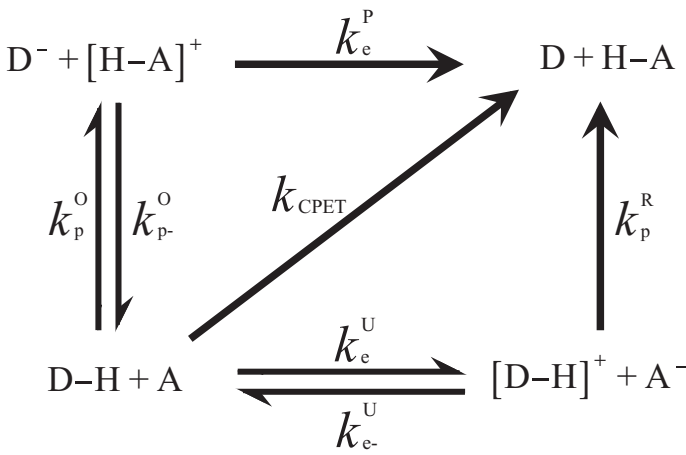

FIG. 1. (a) Schematic illustration of a co-linear PCET reaction, where $D_{e} / D_{p}$ and $\mathrm{A}_{\mathrm{e}} / \mathrm{A}_{\mathrm{p}}$ are the respective donor and acceptor for the electron/proton. (b) Schematic illustration of sequential and concerted PCET reaction mechanisms, indicating the rate constants for the individual charge transfer processes. The sequential mechanism proceeds along the horizontal and vertical edges of the schematic, whereas the concerted mechanism proceeds along the diagonal

broad categories for PCET, there exist a range of coupling regimes that depend on the degree of electronic and vibrational non-adiabaticity for the PCET reaction. ${ }^{9-13}$

Rate theories have been derived and successfully employed to study concerted PCET reactions in a variety of limiting regimes, including (i) the fully nonadiabatic regime ${ }^{1,17-19}$ in which the reaction is electronically and vibrationally non-adiabatic, (ii) the partially adiabatic regime $^{10,12,20,21}$ in which the reaction is electronically adiabatic and vibrationally non-adiabatic, and (iii) the fully adi-

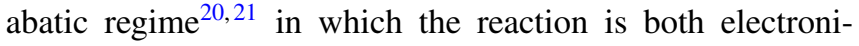
cally and vibrationally adiabatic. These rate theories, which generally employ Golden Rule and linear response approximations, provide a powerful toolkit for investigating both concerted and sequential PCET reactions in many systems. However, the applicability of any given rate theory is limited to the particular coupling regime for which it was derived, and without prior mechanistic information about a given PCET reaction, it can be difficult to know which formulation to apply in practice. Furthermore, with few exceptions, ${ }^{12}$ existing rate theories do not offer scope for the study of PCET reactions with intermediate values for the electronic and vibrational coupling, which exist between the limiting regimes for which the rate theories have been derived. Methods that enable the direct simulation of PCET reactions across all electronic and vibrational coupling regimes, including intermediate regimes, are needed to achieve a unified picture for the dynamics, mechanisms, and driving forces that govern the full range of PCET reactions.

Fundamental theoretical challenges in the description of PCET reactions arise due to the coupling of intrinsically quantum mechanical ET and PT dynamics with slower motions of the surrounding environment. New simulation methods are needed to accurately describe this electron-protonenvironment dynamics and to efficiently and robustly simulate long trajectories that bridge the multiple timescales of these reactions. In this study, we address these challenges by extending the RPMD method to enable the direct simulation of condensed-phase PCET reactions. RPMD ${ }^{22}$ is an approximate quantum dynamical method that is based on Feynman's imaginary-time path integral formulation of statistical mechanics. ${ }^{23,24}$ It provides a classical molecular dynamics model for the real-time evolution of a quantum mechanical system that rigorously preserves detailed balance and samples the quantum Boltzmann distribution. ${ }^{24-26}$ The RPMD method has been previously employed to investigate a wide range of quantized reactive and dynamical processes, ${ }^{27-40}$ ranging from gas-phase triatomic reactions ${ }^{27}$ to enzyme-catalyzed hydrogen tunneling. ${ }^{28}$ We have demonstrated that RPMD simulations can be extended to accurately and efficiently describe coupled electronic and nuclear dynamics in condensed-phase systems, including excess electron diffusion, ${ }^{31}$ injection, ${ }^{32}$ and reactive transfer. ${ }^{33}$ Prior validation of RPMD for the description of ET reactions throughout the normal and activationless regimes, ${ }^{33}$ in combination with prior demonstration of the method for a range of H-transfer processes, ${ }^{27-30}$ provides a basis for expecting the method to adequately describe the dynamics of PCET reactions, which will be tested in the current study.

Alternative theoretical methods have previously advanced our ability to simulate and understand coupled electronic and nuclear dynamics, ${ }^{41-52}$ and promising new methods continue to be introduced. ${ }^{53}$ Established methods include Ehrenfest dynamics, ${ }^{41,42}$ mixed quantum-classical trajectory surface hopping dynamics, ${ }^{43-48}$ the ab initio multiple spawning approach, ${ }^{49}$ and semiclassical methods based on the Meyer-Miller-Stock-Thoss mapping. ${ }^{50-52}$ However, despite their successes, these methods do not yield a dynamics that rigorously preserves detailed balance, ${ }^{54,55}$ a feature that is valuable for the robust calculation of thermal reaction rates $^{56,57}$ and for the utilization of rare-event sampling methods. ${ }^{58,59}$ Although it is clear that other methods must be part of the toolkit for understanding PCET reactions, we emphasize that the formal properties of the RPMD method are ideally suited to this goal.

In this paper, we extend the RPMD method to allow for direct simulation of co-linear, condensed-phase PCET reactions across a wide range of physically relevant regimes. In addition to providing validation for the simulation method via extensive comparison with existing PCET rate theories, we analyze the RPMD reactive trajectories to elucidate a variety of mechanisms for the concerted charge-transfer process. The presented analysis offers a unifying picture for PCET across a wide range of physical regimes, and it suggests new PCET regimes that have yet to be characterized.

\section{RING POLYMER MOLECULAR DYNAMICS}

The RPMD equations of motion for $N$ particles that are quantized using $n$ ring-polymer beads are ${ }^{22,31}$ 


$$
\begin{aligned}
\dot{v}_{i}^{(\alpha)}= & \omega_{n}^{2}\left(q_{i}^{(\alpha+1)}+q_{i}^{(\alpha-1)}-2 q_{i}^{(\alpha)}\right) \\
& -\frac{1}{m_{i}} \frac{\partial}{\partial q_{i}^{(\alpha)}} U\left(q_{1}^{(\alpha)}, q_{2}^{(\alpha)}, \ldots, q_{N}^{(\alpha)}\right),
\end{aligned}
$$

where $v_{i}^{(\alpha)}$ and $q_{i}^{(\alpha)}$ are the velocity and position of the $\alpha$ th bead for the $i$ th particle, respectively, and $q_{i}^{(0)}=q_{i}^{(n)}$. The physical mass for particle $i$ is given by $m_{i}, \omega_{n}=n /(\beta \hbar)$ is the intra-bead harmonic frequency, and $\beta=\left(k_{\mathrm{B}} T\right)^{-1}$ is the reciprocal temperature. The potential energy function of the system is given by $U\left(q_{1}, \ldots, q_{N}\right)$.

To allow for the straightforward comparison with PCET rate theories, we quantize only the transferring electron and proton in this study and consider the classical (i.e., 1-bead) limit for the $N$ solvent degrees of freedom. Furthermore, we employ a mixed-bead-number path-integral representation that reduces the cost of the potential energy surface calculations by utilizing the more rapid convergence of the pathintegral distribution for heavier particles. ${ }^{60}$ We thus obtain the modified RPMD equations of motion:

$$
\begin{aligned}
\dot{v}_{\mathrm{e}}^{(\alpha)}= & \omega_{n_{\mathrm{e}}}^{2}\left(q_{\mathrm{e}}^{(\alpha+1)}+q_{\mathrm{e}}^{(\alpha-1)}-2 q_{\mathrm{e}}^{(\alpha)}\right) \\
& -\frac{1}{m_{\mathrm{e}}} \frac{\partial}{\partial q_{\mathrm{e}}^{(\alpha)}} U\left(q_{\mathrm{e}}^{(\alpha)}, q_{\mathrm{p}}^{\left((\alpha-k) \frac{1}{n_{\mathrm{ep}}}+1\right)}, \mathbf{Q}\right), \\
\dot{v}_{\mathrm{p}}^{(\gamma)}= & \omega_{n_{\mathrm{p}}}^{2}\left(q_{\mathrm{p}}^{(\gamma+1)}+q_{\mathrm{p}}^{(\gamma-1)}-2 q_{\mathrm{p}}^{(\gamma)}\right) \\
& -\frac{1}{m_{\mathrm{p}}} \sum_{l=1}^{n_{\mathrm{ep}}} \frac{\partial}{\partial q_{\mathrm{p}}^{(\gamma)}} U\left(q_{\mathrm{e}}^{\left((\gamma-1) n_{\mathrm{ep}}+l\right)}, q_{\mathrm{p}}^{(\gamma)}, \mathbf{Q}\right),
\end{aligned}
$$

and

$$
\dot{V}_{j}=-\frac{1}{n_{\mathrm{e}} M_{j}} \sum_{\gamma=1}^{n_{\mathrm{p}}} \sum_{l=1}^{n_{\mathrm{ep}}} \frac{\partial}{\partial Q_{j}} U\left(q_{\mathrm{e}}^{\left((\gamma-1) n_{\mathrm{ep}}+l\right)}, q_{\mathrm{p}}^{(\gamma)}, \mathbf{Q}\right),
$$

where $n_{e}$ is the number of imaginary-time ring-polymer beads for the transferring electron, $m_{\mathrm{e}}$ is the physical mass for the electron, and $q_{\mathrm{e}}^{(\alpha)}$ and $v_{\mathrm{e}}^{(\alpha)}$ are the respective position and velocity for the $\alpha$ th ring-polymer bead of the electron; the corresponding quantities for the transferring proton are indicated using subscript "p." In Eqs. (2)-(4), it is assumed that $n_{\mathrm{ep}}=n_{\mathrm{e}} / n_{\mathrm{p}}$ is an integer number, and

$$
k=\alpha-n_{\mathrm{ep}}\left\lfloor\frac{\alpha-1}{n_{\mathrm{ep}}}\right\rfloor,
$$

where $\lfloor\ldots\rfloor$ denotes the floor function. As before, the periodic constraint of the ring-polymer is satisfied via $q_{\mathrm{e}}^{(0)}=q_{\mathrm{e}}^{\left(n_{\mathrm{e}}\right)}$ and $q_{\mathrm{p}}^{(0)}=q_{\mathrm{p}}^{\left(n_{\mathrm{p}}\right)}$, and the intra-bead harmonic frequencies are $\omega_{n_{\mathrm{e}}}=n_{\mathrm{e}} /(\beta \hbar)$ and $\omega_{n_{\mathrm{p}}}=n_{\mathrm{p}} /(\beta \hbar)$. The position, velocity, and mass for the $j$ th classical solvent degree of freedom are given by $Q_{j}, V_{j}$, and $M_{j}$, respectively, and $\mathbf{Q}=\left\{Q_{1}, \ldots, Q_{N}\right\}$.

In the limit of a large number of ring-polymer beads, the RPMD equations of motion yield a time-reversible molecular dynamics that preserves the exact quantum mechanical Boltzmann distribution. ${ }^{24-26}$ Equations (2)-(4) introduce no approximation to Eq. (1) beyond taking the classical limit of the solvent degrees of freedom.
Analogous to the classical thermal rate constant, ${ }^{61-63}$ the RPMD thermal rate constant can be expressed as ${ }^{56,57}$

$$
k_{\mathrm{RPMD}}=\lim _{t \rightarrow \infty} \kappa(t) k_{\mathrm{TST}},
$$

where $k_{\mathrm{TST}}$ is the transition state theory (TST) estimate for the rate associated with the dividing surface $\xi(\mathbf{r})=\xi^{\ddagger}, \xi(\mathbf{r})$ is a collective variable that distinguishes between the reactant and product basins of stability, and $\kappa(t)$ is the time-dependent transmission coefficient that accounts for recrossing of trajectories through the dividing surface. We have introduced $\mathbf{r}=\left\{q_{\mathrm{e}}^{(1)}, \ldots, q_{\mathrm{e}}^{\left(n_{\mathrm{e}}\right)}, q_{\mathrm{p}}^{(1)}, \ldots q_{\mathrm{p}}^{\left(n_{\mathrm{p}}\right)}, Q_{1}, \ldots Q_{N}\right\}$ to denote the position vector for the full system in the ring-polymer representation. As is the case for both exact classical and exact quantum dynamics, the RPMD method yields reaction rates and mechanisms that are independent of the choice of dividing surface. ${ }^{56,57,64}$

The TST rate in Eq. (6) is calculated using $29,33,65,66$

$$
k_{\mathrm{TST}}=(2 \pi \beta)^{-1 / 2}\left\langle g_{\xi}\right\rangle_{\mathrm{c}} \frac{e^{-\beta \Delta F\left(\xi^{\ddagger}\right)}}{\int_{-\infty}^{\xi^{\ddagger}} d \xi e^{-\beta \Delta F(\xi)}},
$$

where $F(\xi)$ is the free energy (FE) along $\xi$,

$$
e^{-\beta \Delta F(\xi)}=\frac{\langle\delta(\xi(\mathbf{r})-\xi)\rangle}{\left\langle\delta\left(\xi(\mathbf{r})-\xi_{r}\right)\right\rangle}
$$

$\xi_{r}$ is a reference point in the reactant basin, and ${ }^{29,67-69}$

$$
g_{\xi}(\mathbf{r})=\left[\sum_{i=1}^{d} \frac{1}{m_{i}}\left(\frac{\partial \xi(\mathbf{r})}{\partial r_{i}}\right)^{2}\right]^{1 / 2} .
$$

Here, $r_{i}$ is an element of the position vector $\mathbf{r}, m_{i}$ is the corresponding physical mass, and $d$ is the length of vector $\mathbf{r}$. The equilibrium ensemble average is denoted as

$$
\langle\ldots\rangle=\frac{\int d \mathbf{r} \int d \mathbf{v} e^{-\beta H(\mathbf{r}, \mathbf{v})}(\ldots)}{\int d \mathbf{r} \int d \mathbf{v} e^{-\beta H(\mathbf{r}, \mathbf{v})}},
$$

and the average over the ensemble constrained to the dividing surface is denoted as

$$
\langle\ldots\rangle_{\mathrm{c}}=\frac{\int d \mathbf{r} \int d \mathbf{v} e^{-\beta H(\mathbf{r}, \mathbf{v})}(\ldots) \delta\left(\xi(\mathbf{r})-\xi^{\ddagger}\right)}{\int d \mathbf{r} \int d \mathbf{v} e^{-\beta H(\mathbf{r}, \mathbf{v})} \delta\left(\xi(\mathbf{r})-\xi^{\ddagger}\right)},
$$

where

$$
\begin{aligned}
H(\mathbf{r}, \mathbf{v})= & \sum_{j=1}^{N} \frac{1}{2} M_{j} V_{j}^{2}+\sum_{\alpha=1}^{n_{e}} \frac{1}{2} m_{\mathrm{b}, \mathrm{e}}\left(v_{\mathrm{e}}^{(\alpha)}\right)^{2} \\
& +\sum_{\gamma=1}^{n_{p}} \frac{1}{2} m_{\mathrm{b}, \mathrm{p}}\left(v_{\mathrm{p}}^{(\gamma)}\right)^{2}+U_{\mathrm{RP}}(\mathbf{r}) .
\end{aligned}
$$

Here, $m_{\mathrm{b}, \mathrm{e}}$ and $m_{\mathrm{b}, \mathrm{p}}$ are the fictitious Parrinello-Rahman masses for the electron and proton, respectively, ${ }^{25}$ $\mathbf{v}=\left\{v_{\mathrm{e}}^{(1)}, \ldots, v_{\mathrm{e}}^{\left(n_{\mathrm{e}}\right)}, v_{\mathrm{p}}^{(1)}, \ldots, v_{\mathrm{p}}^{\left(n_{\mathrm{p}}\right)}, V_{1}, \ldots, V_{N}\right\} \quad$ is the velocity vector for the full system in the ring-polymer 
representation, and

$$
\begin{aligned}
U_{\mathrm{RP}}(\mathbf{r})= & \frac{1}{n_{\mathrm{e}}} \sum_{\alpha=1}^{n_{\mathrm{e}}} \frac{1}{2} m_{e} \omega_{n_{\mathrm{e}}}^{2}\left(q_{\mathrm{e}}^{(\alpha)}-q_{\mathrm{e}}^{(\alpha-1)}\right)^{2} \\
& +\frac{1}{n_{\mathrm{p}}} \sum_{\gamma=1}^{n_{\mathrm{p}}} \frac{1}{2} m_{p} \omega_{n_{\mathrm{p}}}^{2}\left(q_{\mathrm{p}}^{(\gamma)}-q_{\mathrm{p}}^{(\gamma-1)}\right)^{2} \\
& +\frac{1}{n_{\mathrm{e}}} \sum_{\gamma=1}^{n_{\mathrm{p}}} \sum_{l=1}^{n_{\mathrm{ep}}} U\left(q_{\mathrm{e}}^{\left((\gamma-1) n_{\mathrm{ep}}+l\right)}, q_{\mathrm{p}}^{(\gamma)}, \mathbf{Q}\right) .
\end{aligned}
$$

The transmission coefficient in Eq. (7) is obtained from the flux-side correlation function, ${ }^{56,57}$

$$
\kappa(t)=\frac{\left\langle\dot{\xi}_{0} h\left(\xi\left(\mathbf{r}_{t}\right)-\xi^{\ddagger}\right)\right\rangle_{\mathrm{c}}}{\left\langle\dot{\xi}_{0} h\left(\dot{\xi}_{0}\right)\right\rangle_{\mathrm{c}}},
$$

by releasing RPMD trajectories from the equilibrium ensemble constrained to the dividing surface. Here, $h(\xi)$ is the Heaviside function, $\dot{\xi}_{0}$ is the time-derivative of the collective variable upon initialization of the RPMD trajectory from the dividing surface with the initial velocities sampled from the Maxwell-Boltzmann (MB) distribution, and $\mathbf{r}_{t}$ is the time-evolved position of the system along the RPMD trajectory.

\section{PCET RATE THEORIES}

A primary focus of this study is to compare the RPMD method with rate theories that have been derived for the various limiting regimes of PCET. We thus summarize these PCET rate theories below.

\section{A. Concerted PCET in the fully adiabatic regime}

For the fully adiabatic regime, both the electronic coupling and vibrational coupling between the concerted PCET reactant and product states are large in comparison to the thermal energy, $k_{\mathrm{B}} T$. The reaction proceeds in the ground vibronic state, and it is appropriately described using the expression of Hynes and co-workers ${ }^{20,21}$

$$
k_{\mathrm{CPET}}^{\mathrm{ad}}=\frac{\omega_{s}}{2 \pi} \exp \left[\frac{-\Delta G_{\mathrm{ad}}^{\ddagger}}{k_{\mathrm{B}} T}\right],
$$

where $\omega_{s}$ is the solvent frequency, $\Delta G_{\mathrm{ad}}^{\ddagger}$ is the free-energy barrier for the reaction calculated from the difference of the ground vibronic energy level at its minimum and at its maximum with respect to the solvent coordinate, and $k_{\mathrm{B}}$ is Boltzmann's constant.

\section{B. Concerted PCET in the partially adiabatic regime}

For the partially adiabatic regime, the electronic coupling is large in comparison to $k_{\mathrm{B}} T$, whereas the vibrational coupling is small in comparison to $k_{\mathrm{B}} T$. The reaction proceeds in the ground electronic state, and it is appropriately described using the expression of Cukier ${ }^{10}$ and Hynes and co- workers, ${ }^{20,21}$

$$
k_{\text {CPET }}^{\mathrm{pad}}=\frac{2 \pi}{\hbar} V_{\mu \nu}^{2}\left(4 \pi \lambda k_{\mathrm{B}} T\right)^{-1 / 2} \exp \left[\frac{-\Delta G^{\ddagger}}{k_{\mathrm{B}} T}\right],
$$

where $\lambda$ is the concerted PCET reorganization energy associated with the transfer of both the electron and proton,

$$
\Delta G^{\ddagger}=\frac{\left(\lambda+\Delta G^{0}\right)^{2}}{4 \lambda k_{\mathrm{B}} T},
$$

$\Delta G^{0}$ is the driving force for the concerted PCET reaction, and $V_{\mu \nu}$ is the vibronic coupling. In this regime, the vibronic coupling is equal to the vibrational coupling, $V_{\mathrm{PT}}$, such that

$$
\begin{aligned}
V_{\mu \nu} & =V_{\mathrm{PT}} \\
& =\frac{E_{1}-E_{0}}{2} .
\end{aligned}
$$

$V_{\mathrm{PT}}$ is obtained from the splitting between the vibrational ground state energy, $E_{0}$, and first excited state energy, $E_{1}$, calculated on the lowest electronic adiabat. Equation (16) assumes that only a single initial and final vibrational states are involved in the concerted PCET reaction. ${ }^{10,20,21}$

\section{Concerted PCET in the fully non-adiabatic regime}

For the fully non-adiabatic regime, both the electronic coupling and vibrational coupling are small in comparison to $k_{\mathrm{B}} T$. The reaction is appropriately described using the expression of Cukier ${ }^{17,18}$ and Hammes-Schiffer and co-workers ${ }^{1,19}$

$k_{\mathrm{CPET}}^{\mathrm{nad}}=\frac{2 \pi}{\hbar} \sum_{\mu} P_{\mu} \sum_{\nu} V_{\mu \nu}^{2}\left(4 \pi \lambda k_{\mathrm{B}} T\right)^{-1 / 2} \exp \left[\frac{-\Delta G_{\mu \nu}^{\ddagger}}{k_{\mathrm{B}} T}\right]$,

where $\mu$ and $v$ index the reactant and product vibrational states, respectively, $P_{\mu}$ is the Boltzmann probability of the reactant vibrational state, and

$$
\Delta G_{\mu \nu}^{\ddagger}=\frac{\left(\lambda+\Delta G^{0}+\epsilon_{v}-\epsilon_{\mu}\right)^{2}}{4 \lambda k_{\mathrm{B}} T},
$$

where $\epsilon_{\mu}$ and $\epsilon_{v}$ are the respective energies of the reactant and product vibrational states relative to their corresponding ground state. In this regime, the vibronic coupling is given by

$$
V_{\mu \nu}=\langle\mu \mid v\rangle V_{\mathrm{ET}},
$$

where $\langle\mu \mid \nu\rangle$ is the overlap between reactant and product vibrational wavefunctions, and $V_{\mathrm{ET}}$ is the electronic coupling.

\section{ET rate theories}

We also compare RPMD simulations with rate theories that correspond to the electronically adiabatic and nonadiabatic regimes for pure ET. These ET rate theories are summarized below.

\section{Adiabatic ET}

For the electronically adiabatic regime, the electronic coupling between the reactant and product ET states is large in comparison to $k_{\mathrm{B}} T$. The reaction proceeds in the 
ground electronic state, and it is appropriately described using Eq. (15), except with the free-energy barrier, $\Delta G_{\text {ad }}^{\ddagger}$, calculated from the difference of the ground electronic energy level at its minimum and at its maximum with respect to the solvent coordinate. $^{70,71}$

\section{Non-adiabatic ET}

For the electronically non-adiabatic regime, the electronic coupling is small in comparison to $k_{\mathrm{B}} T$. The reaction is appropriately described using the standard Marcus theory expression, ${ }^{72-74}$

$$
k_{\mathrm{ET}}^{\mathrm{nad}}=\frac{2 \pi}{\hbar}\left|V_{\mathrm{ET}}\right|^{2}\left(4 \pi \lambda k_{\mathrm{b}} T\right)^{-1 / 2} \exp \left[\frac{-\Delta G^{\ddagger}}{k_{\mathrm{B}} T}\right],
$$

where

$$
\Delta G_{\mu \nu}^{\ddagger}=\frac{\left(\lambda+\Delta G^{0}\right)^{2}}{4 \lambda k_{\mathrm{B}} T} .
$$

Here, $V_{\mathrm{ET}}, \lambda$, and $\Delta G^{0}$ are, respectively, the electronic coupling, reorganization energy, and driving force associated with the ET reaction.

\section{PCET MODEL SYSTEMS}

Throughout this paper, condensed-phase PCET is described using a co-linear system-bath model. The model is expressed in the position representation using the potential energy function

$$
U\left(q_{\mathrm{e}}, q_{\mathrm{p}}, q_{\mathrm{s}}, \mathbf{Q}\right)=U_{\mathrm{sys}}\left(q_{\mathrm{e}}, q_{\mathrm{p}}, q_{\mathrm{s}}\right)+U_{\mathrm{B}}\left(q_{\mathrm{s}}, \mathbf{Q}\right),
$$

where $U_{\mathrm{B}}\left(q_{\mathrm{s}}, \mathbf{Q}\right)$ is the potential energy term associated with the bath coordinates, and

$$
\begin{aligned}
U_{\mathrm{sys}}\left(q_{\mathrm{e}}, q_{\mathrm{p}}, q_{\mathrm{s}}\right)= & U_{\mathrm{e}}\left(q_{\mathrm{e}}\right)+U_{\mathrm{p}}\left(q_{\mathrm{p}}\right)+U_{\mathrm{s}}\left(q_{\mathrm{s}}\right) \\
& +U_{\mathrm{es}}\left(q_{\mathrm{e}}, q_{\mathrm{s}}\right)+U_{\mathrm{ps}}\left(q_{\mathrm{p}}, q_{\mathrm{s}}\right) \\
& +U_{\mathrm{ep}}\left(q_{\mathrm{e}}, q_{\mathrm{p}}\right)
\end{aligned}
$$

is the system potential energy. The scalar coordinates $q_{\mathrm{e}}, q_{\mathrm{p}}$, and $q_{\mathrm{s}}$ describe the positions of the electron, proton, and solvent modes, respectively, and $\mathbf{Q}$ is the vector of bath oscillator positions.

The first term in the system potential energy function models the interaction of the transferring electron with its donor and acceptor sites,

$$
U_{\mathrm{e}}\left(q_{\mathrm{e}}\right)= \begin{cases}a_{\mathrm{D}} q_{\mathrm{e}}^{2}+b_{\mathrm{D}} q_{\mathrm{e}}+c_{\mathrm{D}}, & r_{\mathrm{D}}^{\text {out }} \leq q_{\mathrm{e}} \leq r_{\mathrm{D}}^{\text {in }} \\ a_{\mathrm{A}} q_{\mathrm{e}}^{2}+b_{\mathrm{A}} q_{\mathrm{e}}+c_{\mathrm{A}}, & r_{\mathrm{A}}^{\text {in }} \leq q_{\mathrm{e}} \leq r_{\mathrm{A}}^{\text {out }} \\ -\mu_{\mathrm{e}}\left[\frac{1}{\left|q_{\mathrm{e}}-r_{\mathrm{D}}\right|}+\frac{1}{\left|q_{\mathrm{e}}-r_{\mathrm{A}}\right|}\right], & \text { otherwise }\end{cases}
$$

where $r_{\mathrm{D}}$ and $r_{\mathrm{A}}$ are the positions of the electron donor and acceptor sites. This one-dimensional (1D) potential energy function consists of two symmetric coulombic wells, each of which is capped by quadratic functions to remove singularities.
The second term in the system potential energy function models the interaction between the transferring proton and its donor and acceptor sites,

$$
U_{\mathrm{p}}\left(q_{\mathrm{p}}\right)=-\frac{m_{\mathrm{p}} \omega_{\mathrm{p}}^{2}}{2} q_{\mathrm{p}}^{2}+\frac{m_{\mathrm{p}}^{2} \omega_{\mathrm{p}}^{4}}{16 V_{0}} q_{\mathrm{p}}^{4}
$$

Here, $\omega_{\mathrm{p}}$ is the proton vibrational frequency and $V_{0}$ is the intrinsic PT barrier height.

The next three terms in the system potential energy function model the solvent potential and the electron- and protonsolvent interactions. Specifically,

$$
U_{\mathrm{s}}\left(q_{\mathrm{s}}\right)=\frac{1}{2} m_{\mathrm{s}} \omega_{\mathrm{s}}^{2} q_{\mathrm{s}}^{2},
$$

$$
U_{\mathrm{es}}\left(q_{\mathrm{e}}, q_{\mathrm{s}}\right)=-\mu_{\mathrm{es}} q_{\mathrm{e}} q_{\mathrm{s}}
$$

and

$$
U_{\mathrm{ps}}\left(q_{\mathrm{p}}, q_{\mathrm{s}}\right)=-\mu_{\mathrm{ps}} q_{\mathrm{p}} q_{\mathrm{s}}
$$

where $m_{s}$ is the solvent mass and $\omega_{\mathrm{s}}$ is the effective frequency of the solvent coordinate. The solvent coupling parameters, $\mu_{\mathrm{es}}$ and $\mu_{\mathrm{ps}}$, are of opposite sign due to the opposing charges of the transferring electron and proton.

Interactions between the transferring electron and proton are modeled via the capped coulombic potential:

$$
U_{\mathrm{e}}\left(q_{\mathrm{e}}\right)=\left\{\begin{array}{ll}
-\frac{\mu_{\mathrm{ep}}}{\left|q_{\mathrm{e}}-q_{\mathrm{p}}\right|}, & \left|q_{\mathrm{e}}-q_{\mathrm{p}}\right|>R_{\mathrm{cut}} \\
-\frac{\mu_{\mathrm{ep}}}{R_{\mathrm{cut}}}, & \text { otherwise }
\end{array} .\right.
$$

The potential energy term $U_{\mathrm{B}}\left(q_{\mathrm{s}}, \mathbf{Q}\right)$ models the harmonic bath that is coupled to the PCET reaction. The bath exhibits an ohmic spectral density $J(\omega)$ with cutoff frequency $\omega_{\mathrm{c}},{ }^{75,76}$ such that

$$
J(\omega)=\eta \omega e^{-\omega / \omega_{\mathrm{c}}},
$$

where $\eta$ denotes the friction coefficient. The continuous spectral density is discretized into $f$ oscillators with frequencies: ${ }^{56,77}$

$$
\omega_{j}=-\omega_{\mathrm{c}} \ln \left(\frac{j-0.5}{f}\right)
$$

and coupling constants:

$$
c_{j}=\omega_{j}\left(\frac{2 \eta M \omega_{\mathrm{c}}}{f \pi}\right)^{1 / 2},
$$

such that

$$
U_{\mathrm{B}}\left(q_{\mathrm{s}}, \mathbf{Q}\right)=\sum_{j=1}^{f}\left[\frac{1}{2} M \omega_{j}^{2}\left(Q_{j}-\frac{c_{j} q_{\mathrm{s}}}{M \omega_{j}^{2}}\right)^{2}\right] .
$$

Here, $M$ is the mass of each bath oscillator, and $\omega_{j}$ and $Q_{j}$ are the respective frequency and position for the $j$ th oscillator.

We have developed system parameters to model condensed-phase PCET reactions throughout a range of different physical regimes. Specifically, System 1 models the fully non-adiabatic regime, Systems $2 \mathrm{a}-2 \mathrm{f}$ model the transition between the fully non-adiabatic and partially adiabatic 
regimes, and Systems 3a-3e model the transition between the partially adiabatic and fully adiabatic regimes. Full details of the parameterization are provided in Appendices A and B.

We also employ a system-bath model to investigate pure ET in this study, with a potential energy function:

$$
\begin{aligned}
U^{\mathrm{ET}}\left(q_{\mathrm{e}}, q_{\mathrm{s}}, \mathbf{Q}\right)= & U_{\mathrm{e}}\left(q_{\mathrm{e}}\right)+U_{\mathrm{s}}\left(q_{\mathrm{s}}\right)+U_{\mathrm{es}}\left(q_{\mathrm{e}}, q_{\mathrm{s}}\right) \\
& +U_{\mathrm{B}}\left(q_{\mathrm{s}} \mathbf{Q}\right),
\end{aligned}
$$

that is obtained by simply removing the proton-dependent terms in Eqs. (24) and (25). Systems $4 \mathrm{a}-4 \mathrm{~g}$ model the transition between non-adiabatic and adiabatic ET. Full details of the parameterization for the ET reactions are provided in Appendices A and B.

\section{CALCULATION DETAILS}

Calculations on System 1, Systems 2a-2f, and Systems $4 \mathrm{a}-4 \mathrm{~g}$ are performed at $T=300 \mathrm{~K}$; calculations on Systems $3 \mathrm{a}-3 \mathrm{e}$ are performed at the lower temperature of $T=100 \mathrm{~K}$ to clearly exhibit the transition between the partially adiabatic and fully adiabatic regimes for PCET. For all systems, the harmonic bath is discretized using $f=12$ degrees of freedom.

\section{A. RPMD simulations}

In all simulations, the RPMD equations of motion are evolved using the velocity Verlet algorithm. ${ }^{78}$ As in previous RPMD simulations, each timestep for the electron and proton involves separate coordinate updates due to forces arising from the physical potential and due to exact evolution of the purely harmonic portion of the ring-polymer potentials. ${ }^{79}$ The electron is quantized with $n_{\mathrm{e}}=1024$ ring-polymer beads in all systems, while the proton is quantized with $n_{\mathrm{p}}=32$ ringpolymer beads for Systems 1 and $2 \mathrm{a}-2 \mathrm{f}$ and with $n_{\mathrm{p}}=128$ for Systems 3a-3e. The larger number of beads for Systems 3a-3e is necessary due to the lower temperature.

Two collective variables are used to monitor the PCET reaction mechanism in the RPMD simulations. The progress of the electron is characterized by a "bead-count" coordinate, $f_{\mathrm{b}}$, that reports on the fraction of ring-polymer beads that are located on the electron donor,

$$
f_{\mathrm{b}}\left(q_{\mathrm{e}}^{(1)}, \ldots, q_{\mathrm{e}}^{\left(n_{\mathrm{e}}\right)}\right)=\frac{1}{n_{\mathrm{e}}} \sum_{\alpha=1}^{n_{\mathrm{e}}} \tanh \left(\phi q_{\mathrm{e}}^{(\alpha)}\right),
$$

where $\phi=-3 \cdot 0 / r_{\mathrm{D}}$. The progress of the proton is characterized using the ring-polymer centroid in the proton position coordinate,

$$
\bar{q}_{\mathrm{p}}\left(q_{\mathrm{p}}^{(1)}, \ldots, q_{\mathrm{p}}^{\left(n_{\mathrm{p}}\right)}\right)=\frac{1}{n_{\mathrm{p}}} \sum_{\gamma=1}^{n_{\mathrm{p}}} q_{\mathrm{p}}^{(\gamma)} .
$$

\section{RPMD rate calculations for concerted PCET}

The RPMD reaction rate is calculated from the product of the TST rate and the transmission coefficient (Eq. (6)). The FE profiles that appear in the TST rate expres- sion (Eq. (7)) are obtained using umbrella sampling and the weighted histogram analysis method (WHAM), as described below. $^{80,81}$

For System 2f, the 1D FE profile used in the rate calculation is obtained in the proton centroid coordinate, $F\left(\bar{q}_{\mathrm{p}}\right)$, using the following umbrella sampling protocol. Nine independent sampling trajectories are harmonically restrained to uniformly spaced values of $\bar{q}_{\mathrm{p}}$ in the region $\left[-0.20 a_{0}, 0.20 a_{0}\right.$ ] using a force constant of 1.3 a.u. Additionally, 18 independent sampling trajectories are harmonically restrained to uniformly spaced values of $\bar{q}_{\mathrm{p}}$ in both the region $\left[-1.10 a_{0},-0.25 a_{0}\right]$ and in $\left[0.25 a_{0}, 1.10 a_{0}\right]$ using a lower force constant of 1.0 a.u. to ensure extensive overlap among the sampled distributions. The equilibrium sampling trajectories are performed using path-integral molecular dynamics (PIMD) with $m_{\mathrm{b}, \mathrm{e}}=2000$ a.u. and $m_{\mathrm{b}, \mathrm{p}}=1836.1$ a.u., which allows for a timestep of $0.1 \mathrm{fs}$. Each sampling trajectory is run for $10 \mathrm{~ns}$, and thermostatting is performed by re-sampling the velocities from the MB distribution every $500 \mathrm{fs}$. We note that this choice of the Parrinello-Rahman masses, $m_{\mathrm{b}, \mathrm{e}}$ and $m_{\mathrm{b}, \mathrm{p}}$, allows for a large timestep in the sampling trajectories but has no affect on $F\left(\bar{q}_{p}\right)$ or any other equilibrium ensemble average. $^{25,26}$

For all PCET systems other than System $2 f$, the $1 D$ FE profile used in the rate calculation is obtained in the electron bead-count coordinate, $F\left(f_{\mathrm{b}}\right)$, using the following umbrella sampling protocol. Ninety-three independent sampling trajectories are harmonically restrained to uniformly spaced values of $f_{\mathrm{b}}$ in the region $[-0.92,0.92]$ using a force constant of 20 a.u.; seven independent sampling trajectories are harmonically restrained to uniformly spaced values of $f_{\mathrm{b}}$ in both the region $[-0.991,-0.985]$ and in $[0.985,0.991]$ using a higher force constant of 5000 a.u.; nine independent sampling trajectories are harmonically restrained to uniformly spaced values of $f_{\mathrm{b}}$ in both the region $[-1.0,-0.992]$ and in $[0.992,1.0]$ using a higher force constant of 10000 a.u.; 32 independent sampling trajectories are harmonically restrained to the values of $f_{\mathrm{b}} \in\{ \pm 0.93, \pm 0.935, \pm 0.94, \pm 0.945, \pm 0.95, \pm 0.955$, $\pm 0.96, \pm 0.962, \pm 0.965, \pm 0.967, \pm 0.97, \pm 0.974, \pm 0.976$, $\pm 0.978, \pm 0.98, \pm 0.982\}$ using a force constant of 500 a.u. For Systems 1 and 2a-2e, an auxiliary restraining potential is introduced for the PIMD sampling trajectories to restrict the system to the concerted channel, as described in Appendix C. Each sampling trajectory is run for $10 \mathrm{~ns}$ using a timestep of 0.1 fs, with $m_{\mathrm{b}, \mathrm{e}}=2000$ a.u. and $m_{\mathrm{b}, \mathrm{p}}=1836.1$ a.u. Thermostatting is performed by re-sampling the velocities from the MB distribution every 500 fs.

For System 2f, the transmission coefficient (Eq. (14)) is calculated using RPMD trajectories that are released from the dividing surface associated with $\bar{q}_{\mathrm{p}}=0$. A total of 6000 RPMD trajectories are released. Each RPMD trajectory is evolved for $400 \mathrm{fs}$ using a timestep of $1 \times 10^{-4}$ fs and with the initial velocities sampled from the MB distribution. Initial configurations for the RPMD trajectories are selected every 10 ps from long PIMD sampling trajectories that are constrained to the dividing surface. The sampling trajectories employ $m_{\mathrm{b}, \mathrm{e}}=2000$ a.u., $m_{\mathrm{b}, \mathrm{p}}=1836.1$ a.u., and a timestep of 0.1 fs. Thermostatting is performed by re-sampling the velocities from the MB distribution every 
$500 \mathrm{fs}$. The sampling trajectories are constrained to the dividing surface using the RATTLE algorithm. ${ }^{82}$

For all PCET systems other than System 2f, the transmission coefficient is calculated using RPMD trajectories that are released from the dividing surface associated with $f_{\mathrm{b}}=0$. A total of 4500 RPMD trajectories are released for Systems 1 and $2 \mathrm{a}-2 \mathrm{e}$, and at least 10000 trajectories are released for Systems 3a-3e. Each RPMD trajectory is evolved for $300 \mathrm{fs}$ using a timestep of $1 \times 10^{-4}$ fs and with the initial velocities sampled from the MB distribution. Initial configurations for the RPMD trajectories are selected every $10 \mathrm{ps}$ from long PIMD sampling trajectories that are constrained to the dividing surface. The sampling trajectories employ $m_{\mathrm{b}, \mathrm{e}}=2000$ a.u., $m_{\mathrm{b}, \mathrm{p}}=1836.1$ a.u., and a timestep of $0.1 \mathrm{fs}$. Thermostatting is performed by re-sampling the velocities from the MB distribution every $500 \mathrm{fs}$. The sampling trajectories are constrained to the dividing surface using the RATTLE algorithm. For Systems 1 and 2a-2e, the same auxiliary restraining potential used in the calculation of $F\left(f_{\mathrm{b}}\right)$ is introduced for the PIMD sampling trajectories to restrict the system to the concerted channel, as described in Appendix C; throughout this paper, the RPMD trajectories used to calculate the transmission coefficients are not subjected to auxiliary restraining potentials.

\section{RPMD rate calculations for ET prior to PT}

For System 1, we calculate the rate for both the sequential and concerted PCET mechanisms. For the ET step in the sequential mechanism, we calculate the forward and reverse ET reaction rates between the OU and RU species $\left(k_{\mathrm{e}}^{\mathrm{U}}\right.$ and $k_{\mathrm{e}^{-}}^{\mathrm{U}}$, Fig. 1(b)). The symmetry of the system requires that $k_{\mathrm{e}}^{\mathrm{P}}=k_{\mathrm{e}^{-}}^{\mathrm{U}}$. The 1D FE profile used in the rate calculation for the ET reactions is obtained in the electron bead-count coordinate, $F_{\mathrm{SET}}\left(f_{\mathrm{b}}\right)$, using the same umbrella sampling protocol described for the calculation of $F\left(f_{\mathrm{b}}\right)$; however, in the calculation of $F_{\mathrm{SET}}\left(f_{\mathrm{b}}\right)$, an auxiliary restraining potential is introduced for the PIMD sampling trajectories to restrict the system to the ET channel, as described in Appendix C. The independent sampling trajectories used to calculate $F_{\mathrm{SET}}\left(f_{\mathrm{b}}\right)$ are each run for $15 \mathrm{~ns}$.

The transmission coefficients (Eq. (14)) for the forward and reverse ET reactions are calculated using RPMD trajectories that are released from the dividing surface associated with $f_{\mathrm{b}}=0.18$. A total of 12000 RPMD trajectories are released. Each RPMD trajectory is evolved for 300 fs using a timestep of $1 \times 10^{-4}$ fs and with the initial velocities sampled from the MB distribution. Initial configurations for the RPMD trajectories are selected every 10 ps from long PIMD sampling trajectories that are constrained to the dividing surface. The sampling trajectories employ $m_{\mathrm{b}, \mathrm{e}}=2000$ a.u., $m_{\mathrm{b}, \mathrm{p}}=1836.1$ a.u., and a timestep of 0.1 fs. Thermostatting is performed by re-sampling the velocities from the $\mathrm{MB}$ distribution every $500 \mathrm{fs}$. The sampling trajectories are constrained to the dividing surface using the RATTLE algorithm. The same auxiliary restraining potential used in the calculation of $F_{\mathrm{SET}}\left(f_{\mathrm{b}}\right)$ is introduced for the PIMD sampling trajectories to restrict the system to the ET channel, as described in Appendix C.

\section{RPMD rate calculations for $P T$ prior to $E T$}

For the PT step in the sequential mechanism in System 1, we calculate the forward and reverse PT reactions between the OU and OP species $\left(k_{\mathrm{p}}^{\mathrm{O}}\right.$ and $k_{\mathrm{p}^{-}}^{\mathrm{O}}$, Fig. 1(b)). The symmetry of the system requires that $k_{\mathrm{p}}^{\mathrm{R}}=k_{\mathrm{p}^{-}}^{\mathrm{O}}$. The $1 \mathrm{D}$ FE profile used in the rate calculation for the forward and reverse PT reactions is obtained in the proton centroid coordinate, $F_{\mathrm{SPT}}\left(\bar{q}_{\mathrm{p}}\right)$, using the same umbrella sampling protocol described for the calculation of $F\left(\bar{q}_{\mathrm{p}}\right)$.

The transmission coefficients (Eq. (14)) for the forward and reverse PT reactions are calculated using RPMD trajectories that are released from the dividing surface associated with $\bar{q}_{\mathrm{p}}=0.21 a_{0}$. A total of 10500 RPMD trajectories are released. Each RPMD trajectory is evolved for $300 \mathrm{fs}$ with a timestep of $1 \times 10^{-4}$ fs and with the initial velocities sampled from the MB distribution. Initial configurations for the RPMD trajectories are selected every 10 ps from long PIMD sampling trajectories that are constrained to the dividing surface. The sampling trajectories employ $m_{\mathrm{b}, \mathrm{e}}=2000$ a.u., $m_{\mathrm{b}, \mathrm{p}}=1836.1$ a.u., and a timestep of $0.1 \mathrm{fs}$. Thermostatting is performed by re-sampling the velocities from the MB distribution every $500 \mathrm{fs}$. The sampling trajectories are constrained to the dividing surface using the RATTLE algorithm.

\section{Two-dimensional FE profiles}

For the purpose of analysis, we calculate the twodimensional (2D) FE profile for System 1 in the electron bead-count and proton centroid coordinates, $F\left(f_{\mathrm{b}}, \bar{q}_{\mathrm{p}}\right)$. The 2D FE profile is constructed using PIMD sampling trajectories that are harmonically restrained in both the $f_{\mathrm{b}}$ and $\bar{q}_{\mathrm{p}}$ coordinates. A total of 4553 sampling trajectories are performed, in which the coordinates $f_{\mathrm{b}}$ and $\bar{q}_{\mathrm{p}}$ are sampled using a square grid. The coordinate $f_{\mathrm{b}}$ is sampled using 93 windows that are harmonically restrained to uniformly spaced values of $f_{\mathrm{b}}$ in the region $[-0.92,0.92]$ using a force constant of 20 a.u.; seven windows are harmonically restrained to uniformly spaced values of $f_{\mathrm{b}}$ in both the region $[-0.991$, $-0.985]$ and in [0.985, 0.991] using a higher force constant of 5000 a.u.; nine windows are harmonically restrained to uniformly spaced values of $f_{\mathrm{b}}$ in both the region $[-1.0,-0.992]$ and in $[0.992,1.0]$ using a higher force constant of 10000 a.u.; 32 windows are harmonically restrained to the values of $f_{\mathrm{b}} \in\{ \pm 0.93, \pm 0.935, \pm 0.94, \pm 0.945, \pm 0.95, \pm 0.955, \pm 0.96$, $\pm 0.962, \pm 0.965, \pm 0.967, \pm 0.97, \pm 0.974, \pm 0.976, \pm 0.978$, $\pm 0.98, \pm 0.982\}$ using a force constant of 500 a.u. For each value of $f_{\mathrm{b}}$, the coordinate $\bar{q}_{\mathrm{p}}$ is sampled using nine windows that are harmonically restrained to uniformly spaced values of $\bar{q}_{\mathrm{p}}$ in the region $\left[-0.20 a_{0}, 0.20 a_{0}\right]$ using a force constant of 1.3 a.u., and 10 windows that are harmonically restrained to uniformly spaced values of $\bar{q}_{\mathrm{p}}$ in both the region $\left[-0.70 a_{0}\right.$, $\left.-0.25 a_{0}\right]$ and in $\left[0.25 a_{0}, 1.10 a_{0}\right]$ using a lower force constant of 1.0 a.u. No auxiliary restraining potentials are employed for the calculation of $F\left(f_{\mathrm{b}}, \bar{q}_{\mathrm{p}}\right)$. Each sampling trajectory is run for $2.5 \mathrm{~ns}$ using a timestep of $0.1 \mathrm{fs}$, with $m_{\mathrm{b}, \mathrm{e}}=2000$ a.u. and $m_{\mathrm{b}, \mathrm{p}}=1836.1$ a.u. Thermostatting is performed by re-sampling the velocities from the MB distribution every $500 \mathrm{fs}$. 
We additionally calculate the 2D FE profile for System 1 in the electron bead-count and solvent position coordinates, $F\left(f_{\mathrm{b}}, q_{\mathrm{s}}\right)$, for sampling trajectories corresponding to the concerted PCET reaction. To generate $F\left(f_{\mathrm{b}}, q_{\mathrm{s}}\right)$, the harmonically restrained sampling trajectories used to calculate $F\left(f_{\mathrm{b}}\right)$ for System 1 are utilized.

\section{RPMD transition path ensemble}

As we have done previously, ${ }^{28}$ we analyze the transition path ensemble ${ }^{58}$ for the RPMD trajectories in the current study. Reactive trajectories are generated through forwardand backward-integration of initial configurations drawn from the dividing surface ensemble with initial velocities drawn from the MB distribution. Reactive trajectories correspond to those for which forward- and backward-integrated halftrajectories terminate on opposite sides of the dividing surface. The reactive trajectories that are initialized from the equilibrium Boltzmann distribution on the dividing surface must be reweighted to obtain the unbiased transition path ensemble. ${ }^{58,83,84} \mathrm{~A}$ weighting term, $w_{\alpha}$, is applied to each trajectory, correctly accounting for recrossing and for the fact that individual trajectories are performed in the microcanonical ensemble. This term is given by ${ }^{83}$

$$
w_{\alpha}=\left(\sum_{i}\left|\dot{\xi}(\mathbf{r})_{i}\right|^{-1}\right)^{-1},
$$

where the sum includes all instances in which trajectory $\alpha$ crosses the dividing surface, and $\dot{\xi}(\mathbf{r})_{i}$ is the velocity in the dividing surface collective variable at the $i$ th crossing event. The reweighting has a minor effect on the non-equilibrium averages if the reactive trajectories initialized from the dividing surface exhibit relatively little recrossing, as is the case for the systems studied in this paper. Non-equilibrium averages over the RPMD transition path ensemble are calculated by aligning reactive trajectories at time 0 , defined as the moment in time when the trajectories are released from the dividing surface.

\section{RPMD rate calculations for pure ET}

The RPMD rates for pure ET are calculated for Systems $4 a-4 g$. For Systems 4a-4e, the 1D FE profile used in the rate calculation is obtained in the electron bead-count coordinate, $F_{\text {ET }}\left(f_{\mathrm{b}}\right)$, using the same umbrella sampling protocol described for the calculation of $F\left(f_{\mathrm{b}}\right)$; however, no auxiliary restraining potentials are introduced for the PIMD sampling trajectories.

For Systems 4f and 4g, the 1D FE profile used in the rate calculation is obtained in the solvent coordinate, $F_{\mathrm{ET}}\left(q_{\mathrm{s}}\right)$, by reducing the 2D FE profile in the electron bead-count and solvent coordinates, $F_{\mathrm{ET}}\left(f_{\mathrm{b}}, q_{\mathrm{s}}\right)$. The $2 \mathrm{D}$ FE profile, $F_{\mathrm{ET}}\left(f_{\mathrm{b}}, q_{\mathrm{s}}\right)$, is constructed using PIMD sampling trajectories that are harmonically restrained in both the $f_{\mathrm{b}}$ and $q_{\mathrm{s}}$ coordinates. A total of 5809 sampling trajectories are performed, in which the coordinates $f_{\mathrm{b}}$ and $q_{\mathrm{s}}$ are sampled using a square grid. The coordinate $f_{\mathrm{b}}$ is sampled using 93 windows that are harmonically restrained to uniformly spaced values of $f_{\mathrm{b}}$ in the region $[-0.92,0.92]$ using a force constant of 20 a.u.; seven windows are harmonically restrained to uniformly spaced values of $f_{\mathrm{b}}$ in both the region [ $-0.991,-0.985]$ and in [0.985, 0.991] using a higher force constant of 5000 a.u.; nine windows are harmonically restrained to uniformly spaced values of $f_{\mathrm{b}}$ in both the region $[-1.0,-0.992]$ and in $[0.992,1.0]$ using a higher force constant of 10000 a.u.; 32 windows are harmonically restrained to the values of $f_{\mathrm{b}} \in\{ \pm 0.93, \pm 0.935, \pm 0.94$, $\pm 0.945, \pm 0.95, \pm 0.955, \pm 0.96, \pm 0.962, \pm 0.965, \pm 0.967$, $\pm 0.97, \pm 0.974, \pm 0.976, \pm 0.978, \pm 0.98, \pm 0.982\}$ using a force constant of 500 a.u. For each value of the $f_{\mathrm{b}}$ coordinate, the $q_{\mathrm{s}}$ coordinate is sampled using 37 windows that are harmonically restrained to uniformly spaced values of $q_{\mathrm{s}}$ in the region $\left[-9.0 a_{0}, 9.0 a_{0}\right]$ using a force constant of 0.03 a.u. Each sampling trajectory is run for $2.5 \mathrm{~ns}$ using a timestep of $0.1 \mathrm{fs}$, with $m_{\mathrm{b}, \mathrm{e}}=2000$ a.u. Thermostatting is performed by re-sampling the velocities from the MB distribution every $500 \mathrm{fs}$.

For Systems 4a-4e, the transmission coefficient (Eq. (14)) is calculated using RPMD trajectories that are released from the dividing surface associated with $f_{\mathrm{b}}=0$. A total of 3000 RPMD trajectories are released for Systems 4a-4c, 6000 trajectories for System 4d and 4500 trajectories for System 4e. Each RPMD trajectory is evolved for $300 \mathrm{fs}$ using a timestep of $1 \times 10^{-4}$ fs and with the initial velocities sampled from the MB distribution. Initial configurations for the RPMD trajectories are selected every $10 \mathrm{ps}$ from long PIMD sampling trajectories that are constrained to the dividing surface. The sampling trajectories employ $m_{\mathrm{b}, \mathrm{e}}=2000$ a.u. and a timestep of $0.1 \mathrm{fs}$. Thermostatting is performed by re-sampling the velocities from the MB distribution every $500 \mathrm{fs}$. The sampling trajectories are constrained to the dividing surface using the RATTLE algorithm.

For Systems $4 \mathrm{f}$ and $4 \mathrm{~g}$, the transmission coefficient is calculated using RPMD trajectories that are released from the dividing surface associated with $q_{\mathrm{s}}=0$. A total of 1500 trajectories are released for Systems $4 \mathrm{f}$ and $4 \mathrm{~g}$. Each trajectory is evolved for $700 \mathrm{fs}$ using a timestep of $1 \times 10^{-4} \mathrm{fs}$ and with the initial velocities sampled from the MB distribution. Initial configurations for the RPMD trajectories are selected every $10 \mathrm{ps}$ from long PIMD sampling trajectories that are constrained to the dividing surface. The sampling trajectories employ $m_{\mathrm{b}, \mathrm{e}}=2000$ a.u. and a timestep of $0.1 \mathrm{fs}$. Thermostatting is performed by re-sampling the velocities from the MB distribution every 500 fs. The sampling trajectories are constrained to the dividing surface using the RATTLE algorithm.

\section{B. PCET rate theory calculations}

Expressions for the thermal reaction rates for concerted PCET are provided in Eqs. (15)-(21). Since the current paper considers only symmetric PCET reactions, the driving force, $\Delta G^{0}$, is zero in all cases.

The concerted PCET reorganization energy, $\lambda$, is calculated using the following result for symmetric systems: ${ }^{85-87}$

$$
\lambda=\langle\Delta U\rangle_{\text {reac }},
$$


TABLE I. Values of the electronic coupling, $V_{\mathrm{ET}}$, vibrational coupling, $V_{\mathrm{PT}}$, and reorganization energy, $\lambda$, for the system-bath model systems for PCET. ${ }^{\mathrm{a}}$

\begin{tabular}{lccc}
\hline \hline System & $V_{\mathrm{ET}}$ & $V_{\mathrm{PT}}$ & $\lambda$ \\
\hline 1 & $5.0 \times 10^{-6}$ & $1.8 \times 10^{-6}$ & $1.84 \times 10^{-2}$ \\
$2 \mathrm{a}$ & $5.0 \times 10^{-6}$ & $4.6 \times 10^{-7}$ & $9.71 \times 10^{-3}$ \\
$2 \mathrm{~b}$ & $5.0 \times 10^{-5}$ & $3.6 \times 10^{-7}$ & $9.47 \times 10^{-3}$ \\
$2 \mathrm{c}$ & $5.0 \times 10^{-4}$ & $2.1 \times 10^{-7}$ & $9.78 \times 10^{-3}$ \\
$2 \mathrm{~d}$ & $5.0 \times 10^{-3}$ & $9.6 \times 10^{-8}$ & $9.22 \times 10^{-3}$ \\
$2 \mathrm{e}$ & $2.5 \times 10^{-2}$ & $4.5 \times 10^{-7}$ & $9.32 \times 10^{-3}$ \\
$2 \mathrm{f}$ & $1.0 \times 10^{-1}$ & $4.8 \times 10^{-6}$ & $8.47 \times 10^{-3}$ \\
$3 \mathrm{a}$ & $3.3 \times 10^{-2}$ & $1.5 \times 10^{-8}$ & $3.27 \times 10^{-2}$ \\
$3 \mathrm{~b}$ & $2.7 \times 10^{-2}$ & $2.3 \times 10^{-5}$ & $3.29 \times 10^{-2}$ \\
$3 \mathrm{c}$ & $1.8 \times 10^{-2}$ & $8.5 \times 10^{-4}$ & $3.34 \times 10^{-2}$ \\
$3 \mathrm{~d}$ & $1.5 \times 10^{-2}$ & $2.2 \times 10^{-3}$ & $3.35 \times 10^{-2}$ \\
$3 \mathrm{f}$ & $1.5 \times 10^{-2}$ & $2.8 \times 10^{-3}$ & $3.33 \times 10^{-2}$ \\
\hline \hline
\end{tabular}

${ }^{a}$ All quantities reported in atomic units. For Systems 1 and 2a-2f, $V_{\mathrm{ET}}$ is calculated as described in Sec. V B; for Systems 3a-3f, $V_{\mathrm{ET}}$ is calculated from the splitting between the ground and first-excited adiabatic electronic state energies with $q_{\mathrm{s}}=q_{\mathrm{p}}=0$.

where $\Delta U$ is the concerted PCET energy gap coordinate,

$$
\begin{aligned}
\Delta U= & U_{\mathrm{RP}}\left(-q_{\mathrm{e}}^{(1)}, \ldots,-q_{\mathrm{e}}^{\left(n_{\mathrm{e}}\right)},-q_{\mathrm{p}}^{(1)}, \ldots,-q_{\mathrm{p}}^{\left(n_{\mathrm{p}}\right)}, q_{\mathrm{s}}, \mathbf{Q}\right) \\
& -U_{\mathrm{RP}}\left(q_{\mathrm{e}}^{(1)}, \ldots, q_{\mathrm{e}}^{\left(n_{\mathrm{e}}\right)}, q_{\mathrm{p}}^{(1)}, \ldots, q_{\mathrm{p}}^{\left(n_{\mathrm{p}}\right)}, q_{\mathrm{s}}, \mathbf{Q}\right),
\end{aligned}
$$

and $\langle\ldots\rangle_{\text {reac }}$ denotes the equilibrium ensemble average in the reactant basin. The ensemble average is calculated from a $50 \mathrm{~ns}$ equilibrium PIMD trajectory, where the electron and proton are initialized and remain in the reactant basin. The sampling trajectories employ $m_{\mathrm{b}, \mathrm{e}}=2000$ a.u., $m_{\mathrm{b}, \mathrm{p}}=1836.1$ a.u., and a timestep of $0.1 \mathrm{fs}$. Thermostatting is performed by re-sampling the velocities from the MB distribution every $500 \mathrm{fs}$. Values for the reorganization energy in the various systems are presented in Table I.

The free-energy barrier for PCET in the fully adiabatic regime, $\Delta G_{\text {ad }}^{\ddagger}$ in Eq. (15), is calculated from the difference of the ground vibronic energy level at its minimum and at its maximum with respect to the solvent coordinate. The adiabatic vibronic states are obtained as a function of the solvent coordinate in the range $-4 a_{0} \leq q_{\mathrm{s}} \leq 4 a_{0}$. For each value of $q_{\mathrm{s}}$, the system Hamiltonian associated with $U_{\mathrm{sys}}\left(q_{\mathrm{e}}, q_{\mathrm{p}}, q_{\mathrm{s}}\right)$ (Eq. (25)) is diagonalized using a 2D discrete variable representation (DVR) grid calculation in the electron and proton position coordinates, $q_{\mathrm{e}}$ and $q_{\mathrm{p}}$, respectively. ${ }^{88}$ The grid spans the range $-30 a_{0} \leq q_{\mathrm{e}} \leq 30 a_{0}$ and $-1.5 a_{0} \leq q_{\mathrm{p}} \leq 1.5 a_{0}$, with 1024 and 20 evenly spaced grid points for the electron and proton position, respectively.

The vibronic coupling in the partially adiabatic regime (Eq. (18)) is obtained from the splitting between the ground and first vibrational states calculated for the potential defined by the ground adiabatic electronic state; the ground adiabatic electronic state is calculated for a frozen solvent configuration for which the reactant and product concerted PCET states are degenerate. ${ }^{20,21}$ The calculation of the vibronic coupling in the partially adiabatic regime thus requires two tasks that include $(i)$ the calculation of the adiabatic electronic states as a function of the proton coordinate for a frozen solvent configuration and (ii) the calculation of the proton vibrational states for the potential defined by the lowest adiabatic electronic state. To complete task $(i)$, the adiabatic electronic states are obtained as a function of the proton coordinate in the range $-1.5 a_{0} \leq q_{\mathrm{p}} \leq 1.5 a_{0}$, with $q_{\mathrm{s}}=0$. For each value of $q_{\mathrm{p}}$, the system Hamiltonian is diagonalized using a 1D DVR grid calculation in the electron position coordinate. The grid spans the range $-30 a_{0} \leq q_{\mathrm{e}} \leq 30 a_{0}$ with 2048 evenly spaced grid points. To complete task (ii), a polynomial of the form:

$$
U_{\mathrm{ad}}\left(q_{\mathrm{p}}\right)=\sum_{i=0}^{6} c_{\mathrm{ad}}^{(i)}\left|q_{\mathrm{p}}\right|^{i}
$$

is fit to the lowest adiabatic electronic state in the range $-1.5 a_{0} \leq q_{\mathrm{p}} \leq 1.5 a_{0}$. The vibrational energies, $E_{0}$ and $E_{1}$, are calculated for the fitted potential in Eq. (42) by diagonalizing the 1D DVR Hamiltonian in the proton position coordinate. The grid spans the range $-1.5 a_{0} \leq q_{\mathrm{p}} \leq 1.5 a_{0}$ with 2048 evenly spaced grid points. The values of the vibrational coupling, and hence the partially adiabatic vibronic coupling, are presented in Table I. The coefficients for the polynomial fit to the lowest adiabatic electronic state (Eq. (42)) are presented in Appendix D (Table X).

The vibronic coupling in the fully non-adiabatic regime (Eq. (21)) is obtained from the product of the electronic coupling and the overlap of reactant and product vibrational wavefunctions. The vibrational wavefunctions are calculated for the potential defined by the reactant and product diabatic electronic states; the diabatic electronic states are calculated for a frozen solvent configuration for which the reactant and product concerted PCET states are degenerate. ${ }^{1,17-19}$ The calculation of the vibronic coupling in the fully non-adiabatic regime thus requires three tasks that include $(i)$ the calculation of the electronic coupling, (ii) the calculation of the diabatic electronic states as a function of the proton coordinate for a frozen solvent configuration, and (iii) the calculation of the vibrational energies and wavefunctions for the potential defined by the reactant and product diabatic electronic states. To complete tasks ( $i$ ) and (ii) for Systems 1 and 2a-2f, the electronic coupling and diabatic electronic states are obtained as a function of the proton coordinate for $q_{\mathrm{s}}=0$ using the localization procedure described in Appendix E. The electronic coupling (Eq. (E7)) is found to be nearly constant over the physical range of $q_{\mathrm{p}}$, so we employ a constant value of $V_{\mathrm{ET}}$ that corresponds to the $q_{\mathrm{p}}=0$ value. For Systems $2 \mathrm{e}$ and $2 \mathrm{f}$, the localization procedure does not yield fully localized diabatic states, which contributes to the breakdown of the fully non-adiabatic rate calculation. The values of the electronic coupling are presented in Table I. To complete task (iii), the reactant and product diabatic electronic states (Eqs. (E5) and (E6)) are computed for a uniform grid of 2048 points in the range $-1.5 a_{0} \leq q_{\mathrm{p}} \leq 1.5 a_{0}$, and the reactant and product vibrational energies and wavefunctions are then obtained by diagonalizing the 1D DVR Hamiltonian in the proton position on this grid.

\section{ET rate theory calculations}

Expressions for the thermal reaction rates for ET are provided in Eqs. (15) and (22). The free-energy barrier for ET in the electronically adiabatic regime, $\Delta G_{\text {ad }}^{\ddagger}$ in Eq. (15), is 
TABLE II. Values of the electronic coupling, $V_{\mathrm{ET}}$ and reorganization energy, $\lambda$, for ET systems that vary between the adiabatic and non-adiabatic regimes. $^{\mathrm{a}}$

\begin{tabular}{lcc}
\hline \hline System & $V_{\mathrm{ET}}$ & $\lambda$ \\
\hline $4 \mathrm{a}$ & $1 \times 10^{-6}$ & 7.18 \\
$4 \mathrm{~b}$ & $1 \times 10^{-5}$ & 7.45 \\
$4 \mathrm{c}$ & $1 \times 10^{-4}$ & 7.44 \\
$4 \mathrm{~d}$ & $1 \times 10^{-3}$ & 7.37 \\
$4 \mathrm{e}$ & $4 \times 10^{-3}$ & 7.26 \\
$4 \mathrm{f}$ & $1 \times 10^{-2}$ & 7.18 \\
$4 \mathrm{~g}$ & $2 \times 10^{-2}$ & 7.30 \\
\hline \hline
\end{tabular}

${ }^{\mathrm{a}} \lambda$ is given in units of a.u. $\times 10^{-2}$; all other parameters are given in atomic units.

calculated from the difference of the ground electronic energy level at its minimum and at its maximum with respect to the solvent coordinate. The adiabatic electronic states are obtained as a function of the solvent coordinate in the range $-8.0 a_{0} \leq q_{\mathrm{s}} \leq 8.0 a_{0}$. For each value of $q_{\mathrm{s}}$, the system Hamiltonian associated with Eq. (36) is diagonalized using a 1D DVR grid calculation in the electron position coordinate, $q_{\mathrm{e}}$. The grid spans the range $-30.0 a_{0} \leq q_{\mathrm{e}} \leq 30.0 a_{0}$ with 2048 evenly spaced grid points.

The electronic coupling, $V_{\mathrm{ET}}$ in Eq. (22), is obtained from the splitting between the ground, $\varepsilon_{0}\left(q_{\mathrm{s}}\right)$, and first excited, $\varepsilon_{0}\left(q_{\mathrm{s}}\right)$, adiabatic electronic state energies,

$$
V_{\mathrm{ET}}=\frac{1}{2}\left[\varepsilon_{1}\left(q_{\mathrm{s}}=0\right)-\varepsilon_{0}\left(q_{\mathrm{s}}=0\right)\right]
$$

The ET reorganization energy, $\lambda$, is calculated using Eq. (40), ${ }^{85-87}$ where $\Delta U$ is now the ET energy gap coordinate,

$$
\begin{aligned}
\Delta U= & U_{\mathrm{RP}}^{\mathrm{ET}}\left(-q_{\mathrm{e}}^{(1)}, \ldots,-q_{\mathrm{e}}^{\left(n_{\mathrm{e}}\right)}, q_{\mathrm{s}}, \mathbf{Q}\right) \\
& -U_{\mathrm{RP}}^{\mathrm{ET}}\left(q_{\mathrm{e}}^{(1)}, \ldots, q_{\mathrm{e}}^{\left(n_{\mathrm{e}}\right)}, q_{\mathrm{s}}, \mathbf{Q}\right) .
\end{aligned}
$$

The ensemble average is calculated from a 50 ns equilibrium PIMD trajectory, where the electron is initialized and remains in the reactant basin. The sampling trajectories employ $m_{\mathrm{b}, \mathrm{e}}=2000$ a.u. and a timestep of $0.1 \mathrm{fs}$. Thermostatting is performed by re-sampling the velocities from the MB distribution every $500 \mathrm{fs}$. The values of the reorganization energy are presented in Table II.

\section{RESULTS}

The results are presented in two sections. In the first, we analyze the competition between the concerted and sequential reaction mechanisms for PCET. In the second, we study the kinetics and mechanistic features of concerted PCET reactions across multiple coupling regimes, including the fully non-adiabatic (both electronically and vibrationally nonadiabatic), partially adiabatic (electronically adiabatic, but vibrationally non-adiabatic), and fully adiabatic (both electronically and vibrationally adiabatic) limits.

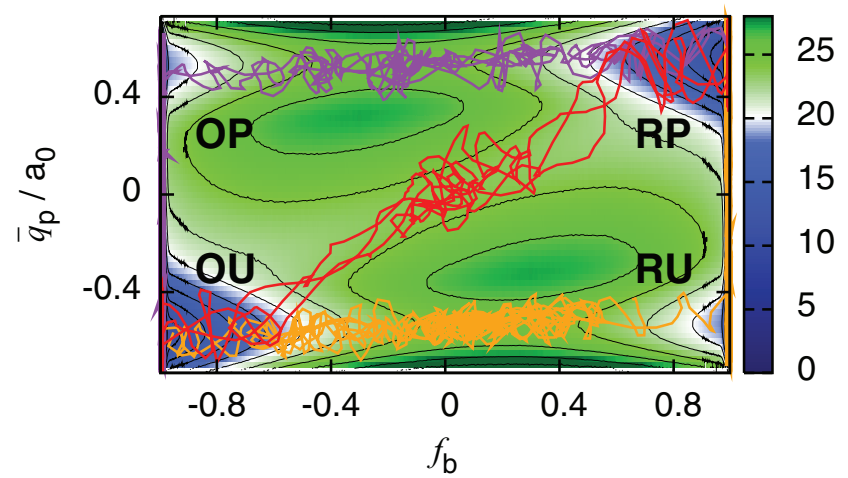

FIG. 2. Reactive RPMD trajectories reveal distinct concerted (red), sequential PT-ET (purple), and sequential ET-PT (orange) reaction mechanisms for PCET in System 1. The trajectories are projected onto the FE surface in the electron bead-count coordinate, $f_{\mathrm{b}}$, and the proton centroid coordinate, $\bar{q}_{\mathrm{p}}$, with contour lines indicating FE increments of $2 \mathrm{kcal} / \mathrm{mol}$.

\section{A. Sequential versus concerted PCET}

We begin by investigating the competing PCET reaction mechanisms in System 1. Figure 2 presents the 2D FE profile for this system along the electron bead-count, $f_{\mathrm{b}}$, and the proton centroid, $\bar{q}_{\mathrm{p}}$ coordinates. The FE profile exhibits four basins of stability corresponding to the various PCET reactant (OU), intermediate (OP and RU), and product (RP) species (Fig. 1(b)). Distinct channels on the FE surface connect the various basins of stability. Due to the symmetry of the reaction, the two channels associated with the PT step of the sequential pathway (connecting OU to OP and RU to RP) are identical, as are the the two channels associated with the ET step of the sequential pathway (connecting OU to RU and OP to RP). A single channel on the FE surface connects OU to $\mathrm{RP}$, bypassing the intermediate species.

Also plotted in Fig. 2 are representative samples from the ensemble of reactive RPMD trajectories for PCET in System 1. The trajectories cluster within the channels on the FE surface, providing a direct illustration of the concerted (red) and sequential (purple and orange) reaction mechanisms for PCET. Such distinct clustering of the reactive trajectories need not be observed in general systems that undergo PCET; we note that the RPMD method makes no a priori assumptions about the preferred reaction mechanism or the existence of distinct sequential and concerted reaction mechanisms for PCET.

We now demonstrate that the concerted PCET mechanisms is dominant in System 1 by computing the RPMD reaction rates for both the concerted and sequential processes. Figures 3(a) and 3(b) illustrate the FE profile and transmission coefficient that together determine the RPMD reaction rate for concerted PCET (Eq. (6)). As was previously found for ET reactions, ${ }^{33}$ the FE profile exhibits a sharp rise as a function of $f_{\mathrm{b}}$ due to the formation of a ring-polymer configuration in which the electron spans the two redox sites (Fig. 3(a), inset), and it exhibits more gradual changes in the range of $\left|f_{\mathrm{b}}\right|<0.97$ due to solvent polarization. For the dividing surface $f_{\mathrm{b}}=0$, the transmission coefficient plateaus at a value of approximately 0.1 , indicating that $f_{\mathrm{b}}$ is a reasonably good reaction coordinate for the process. These 

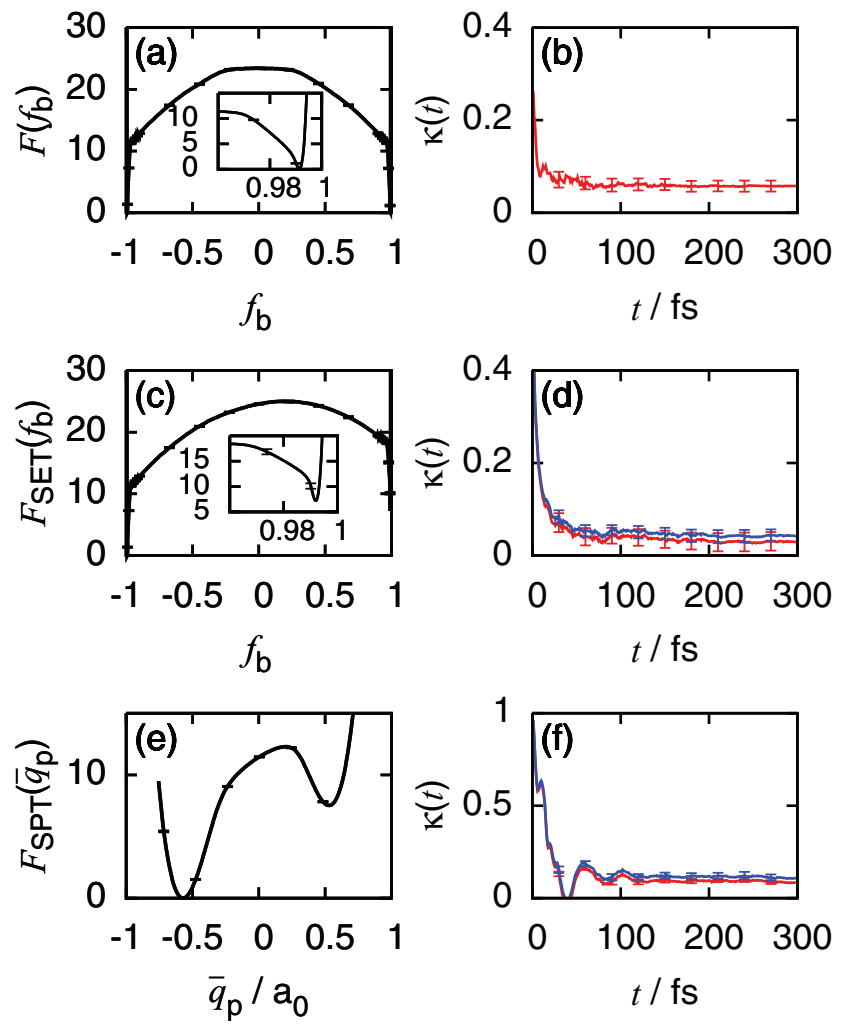

FIG. 3. (a) The 1D FE profile in the electron bead-count coordinate, $F\left(f_{\mathrm{b}}\right)$, utilized in the RPMD rate calculation for the concerted PCET reaction. (b) The corresponding transmission coefficient for the concerted PCET reaction. (c) The $1 \mathrm{D}$ FE profile in the electron bead-count coordinate, $F_{\mathrm{SET}}\left(f_{\mathrm{b}}\right)$, utilized in the RPMD rate calculation for the ET reactions prior to PT in the sequential PCET mechanism. (d) The corresponding forward (red) and reverse (blue) transmission coefficients for the ET reactions prior to PT. (e) The 1D FE profile in the proton centroid coordinate, $F_{\mathrm{SPT}}\left(\bar{q}_{\mathrm{p}}\right)$, utilized in the RPMD rate calculation for the PT reactions prior to ET in the sequential PCET mechanism. (f) The corresponding forward (red) and reverse (blue) transmission coefficients for the PT reactions prior to ET. All FE profiles are plotted in $\mathrm{kcal} / \mathrm{mol}$.

results combine to yield a RPMD rate of $k_{\mathrm{CPET}}=(2.1 \pm 0.7)$ $\times 10^{-20}$ a.u. for the concerted reaction mechanism in System 1.

Figures 3(c)-3(f) present the components of the RPMD rate calculation for the sequential PCET reaction mechanism in System 1. For the ET step of the sequential mechanism, Figs. 3(c) and 3(d) report the FE profile in the electron beadcount coordinate and the forward (red) and reverse (blue) transmission coefficients associated with $f_{\mathrm{b}}=0.18$. For the PT step of the sequential mechanism, Figs. 3(e) and 3(f) report the FE profile in the proton centroid coordinate and the forward (red) and reverse (blue) transmission coefficients associated with $\bar{q}_{\mathrm{p}}=0.21 a_{0}$. The oscillations observed in $\kappa(t)$ for the PT step correspond to the vibrational motion of the transferring proton. These results combine to yield the RPMD rates for the various individual steps in the sequential PCET reaction (Table III).

For the reaction mechanism that involves sequential ET followed by PT, the reaction rate is given by ${ }^{1}$

$$
k_{\mathrm{ep}}=k_{\mathrm{e}}^{\mathrm{U}} \frac{k_{\mathrm{p}}^{\mathrm{R}}}{k_{\mathrm{p}}^{\mathrm{R}}+k_{\mathrm{e}^{-}}^{\mathrm{U}}},
$$

TABLE III. RPMD rates for the forward and reverse ET and PT reactions in the sequential mechanism.

\begin{tabular}{ll}
\hline \hline Rate constant & \\
\hline$k_{\mathrm{e}}^{\mathrm{U}}$ & $(3.6 \pm 2.6) \times 10^{-21}$ \\
$k_{\mathrm{e}^{-}}^{\mathrm{U}}$ & $(1.1 \pm 0.4) \times 10^{-15}$ \\
$k_{\mathrm{e}}^{\mathrm{P}}$ & $(1.1 \pm 0.4) \times 10^{-15}$ \\
$k_{\mathrm{p}}^{\mathrm{O}}$ & $(2.9 \pm 0.3) \times 10^{-13}$ \\
$k_{\mathrm{p}^{-}}^{\mathrm{O}}$ & $(9.6 \pm 1.7) \times 10^{-8}$ \\
$k_{\mathrm{p}}^{\mathrm{R}}$ & $(9.6 \pm 1.7) \times 10^{-8}$ \\
\hline
\end{tabular}

${ }^{a}$ All rates are given in atomic units. The notation for the rate constants is defined in Fig. 1(b).

which numerically yields $k_{\mathrm{ep}}=(3.6 \pm 2.6) \times 10^{-21}$ a.u. Similarly, for the reaction mechanism involving sequential PT followed by ET, the reaction rate is given by ${ }^{1}$

$$
k_{\mathrm{pe}}=k_{\mathrm{p}}^{\mathrm{O}} \frac{k_{\mathrm{e}}^{\mathrm{P}}}{k_{\mathrm{e}}^{\mathrm{P}}+k_{\mathrm{p}^{-}}^{\mathrm{O}}},
$$

which numerically yields $k_{\mathrm{pe}}=(3.4 \pm 1.4) \times 10^{-21}$ a.u. The computed values for $k_{\mathrm{ep}}$ and $k_{\mathrm{pe}}$ are equal to within statistical error, as is consistent with microscopic reversibility in this symmetric system.

Comparison of the reaction rate for the concerted and sequential PCET mechanisms (Table IV) reveals that the reaction rate for the concerted mechanism is approximately six times larger than that of the sequential mechanism; the RPMD method thus predicts that the PCET reaction in System 1 proceeds predominantly via the concerted reaction mechanism. We note that although the reaction dividing surfaces were selected to minimize trajectory recrossing, the rates reported here for the various sequential and concerted steps are rigorously independent of this choice of dividing surface; the mechanistic analysis provided here thus avoids any TST approximations or prior assumptions about the reaction mechanism.

Having established that the concerted mechanism is favored for System 1, we now analyze the RPMD trajectories with respect to the solvent fluctuations and interactions that govern the concerted PCET reaction mechanism.

Figure 4(a) presents the 2D FE profile in the electron bead-count and solvent coordinates, $F\left(f_{\mathrm{b}}, q_{\mathrm{s}}\right)$, computed for the concerted pathway as is described in Sec. V A 4. The FE profile exhibits two basins of stability corresponding to the PCET reactant and product species (OU and RP, respectively), separated by a barrier that corresponds to the dividing surface in the $f_{\mathrm{b}}$ coordinate. Also plotted in Fig. 4(a) are representative samples from the ensemble of reactive RPMD trajectories (red) and the non-equilibrium average over the

TABLE IV. Reaction rates for the full ET-PT, PT-ET, and concerted PCET mechanisms calculated using RPMD and Eqs. (45) and (46). ${ }^{\mathrm{a}}$

\begin{tabular}{ll}
\hline \hline Rate constant & \\
\hline$k_{\text {ep }}$ & $(3.6 \pm 2.6) \times 10^{-21}$ \\
$k_{\text {pe }}$ & $(3.4 \pm 1.4) \times 10^{-21}$ \\
$k_{\text {CPET }}$ & $(2.1 \pm 0.7) \times 10^{-20}$ \\
\hline \hline
\end{tabular}

${ }^{\mathrm{a}}$ All rates are given in atomic units. 
(a)

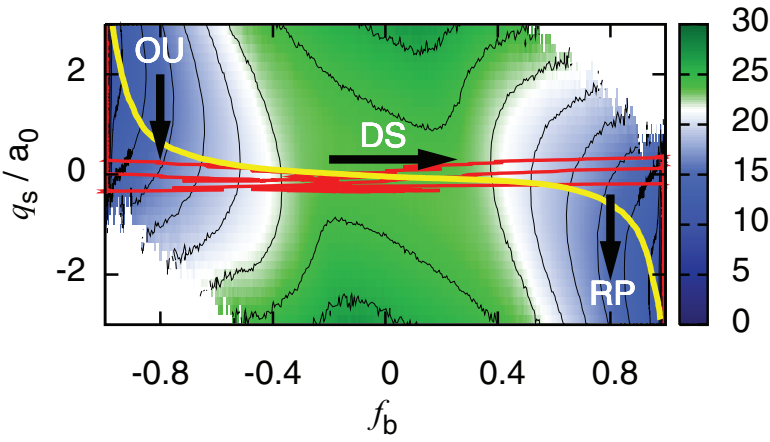

(b)
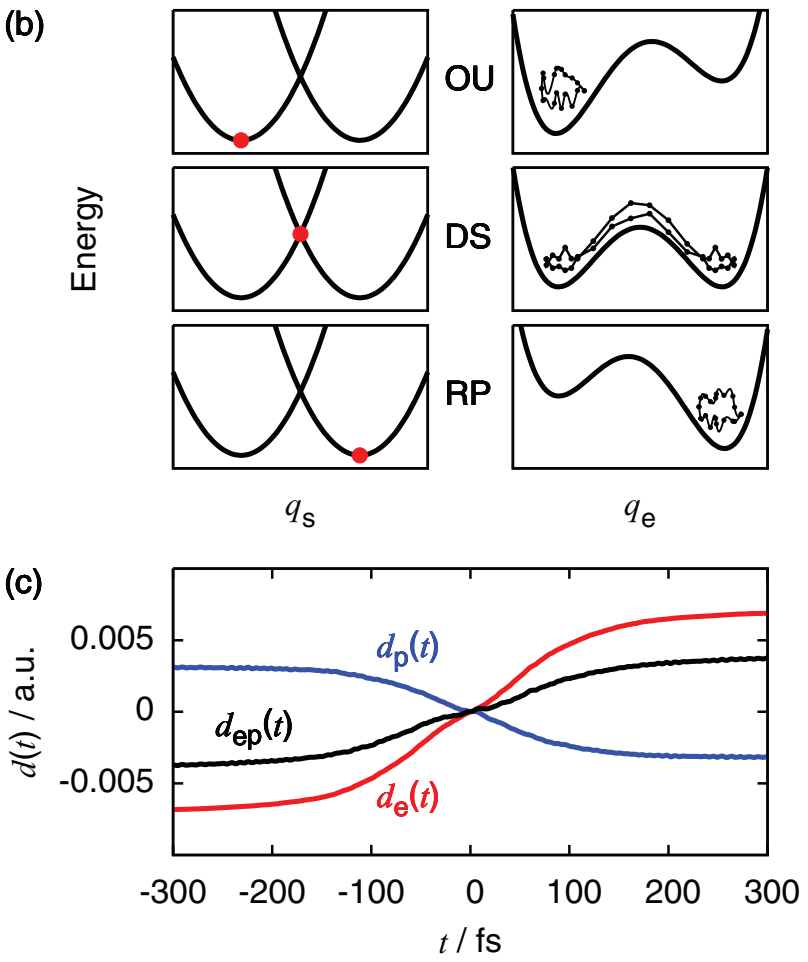

FIG. 4. (a) Reactive RPMD trajectories (red) and the average over the ensemble of reactive trajectories (yellow) for the concerted PCET reaction in System 1 reveal a Marcus-type solvent-gating mechanism indicated by the black arrows. The trajectories are projected onto the FE surface in the electron bead-count coordinate, $f_{\mathrm{b}}$, and the solvent position coordinate, $q_{\mathrm{s}}$, with contour lines indicating FE increments of $2 \mathrm{kcal} / \mathrm{mol}$. The regions corresponding to the concerted PCET reactant (OU), product (RP), and dividing surface (DS) are indicated. (b) Illustration of the mechanism for concerted PCET. The left panels present the vibronic diabatic free energy surfaces along the solvent coordinate; the red dot indicates the solvent configuration associated with the OU, RP, and DS regions indicated in (a). The right panels present the double-well potential that is experienced by the electron in the OU, RP, and DS regions, as well as the ring-polymer configuration in the electron position coordinate at the corresponding points along a typical reactive trajectory. (c) The combined dipole for the transferring particles in the ensemble of reactive RPMD trajectories, $d_{\mathrm{ep}}(t)$ (black), as well as the individual components from the transferring electron, $d_{\mathrm{e}}$ (red), and the transferring proton, $d_{\mathrm{p}}$ (blue), for the concerted PCET reaction in System 1.

ensemble of reactive trajectories (yellow), as described in Sec. V A 5. As was seen for ET, ${ }^{33}$ the reactive RPMD trajectories for concerted PCET follow a Marcus-type solvent-gating mechanism (black arrows), in which solvent reorganization precedes the sudden transfer of both the electron and proton between wells that are nearly degenerate with respect to solvent polarization.
Figure 4(b) elaborates on this mechanism, schematically illustrating the ring-polymer configurations that accompany the various stages of the concerted PCET reaction. In the reactant OU basin, the system rests at the bottom of the solvent potential well for the reactant vibronic diabat (indicated by a red point in the left panel); for this polarized solvent configuration, the transferring electron and proton experience a potential energy surface that favors occupation of the donor sites (shown for the electron position in the right panel). In the dividing surface (DS) region of the concerted PCET reaction, the solvent fluctuation brings the system to configurations at which the vibronic diabats for the transferring electron and proton are nearly degenerate (shown at left), and the transferring particles undergo tunneling between nearly degenerate wells for the donor and acceptor sites (shown at right); also seen in the panel at right is the extended "kink-pair" configuration for the ring-polymer in which the electron spans the two redox sites during the tunneling event. Finally, the figure panels associated with the product RP basin illustrate that as the solvent relaxes to the minimum of the solvent potential well for the product vibronic diabat (left), the transferring electron and proton experience a potential energy surface that favors occupation of the product sites (right). This mechanism observed in the RPMD trajectories is consistent with the mechanisms that are assumed by PCET rate theories in the fully non-adiabatic regime. ${ }^{1,17-19}$

Figure 4(c) illustrates part of the mechanistic basis for the favorability of the concerted PCET reaction in this system. The figure presents the combined dipole for the transferring particles in the ensemble of reactive RPMD trajectories, $d_{\mathrm{ep}}(t)$, as well as the individual components from the transferring electron and proton, $d_{\mathrm{e}}(t)$ and $d_{\mathrm{p}}(t)$, respectively. These terms are computed using

$$
\begin{aligned}
& d_{\mathrm{e}}(t)=-\mu_{\mathrm{es}}\left\langle\bar{q}_{\mathrm{e}}(t)\right\rangle_{\text {traj }}, \\
& d_{\mathrm{p}}(t)=-\mu_{\mathrm{ps}}\left\langle\bar{q}_{\mathrm{p}}(t)\right\rangle_{\text {traj }},
\end{aligned}
$$

and

$$
d_{\mathrm{ep}}(t)=d_{\mathrm{e}}(t)+d_{\mathrm{p}}(t)
$$

where $\bar{q}_{\mathrm{e}}$ and $\bar{q}_{\mathrm{p}}$ are the ring-polymer centroids for the transferring electron and proton, respectively, and $\langle\ldots\rangle_{\text {traj }}$ denotes the non-equilibrium ensemble average over the time-evolved reactive RPMD trajectories for concerted PCET (Sec. V A 5). Figure 4(c) shows that the orientation of $d_{\mathrm{e}}(t)$ and $d_{\mathrm{p}}(t)$ switch during the reaction on similar timescales, which follows from the fact that the two particles are moving both co-linearly and in concert. However, the figure also shows that the magnitude of $d_{\mathrm{ep}}(t)$ is at all times smaller than the larger magnitude of the two component dipoles (i.e., $\left|d_{\mathrm{ep}}(t)\right|<\max \left(\left|d_{\mathrm{e}}(t)\right|,\left|d_{\mathrm{p}}(t)\right|\right)$ ), due to the opposite charge of the two transferring particles. It is thus clear that throughout reactive trajectories for concerted PCET, the degree to which the polar solvent couples to the transferring particles is reduced by the opposing sign of the electron and proton charge. In this sense, the polar solvent creates a driving force for the co-localization of the electron and proton.

We emphasize that although we have previously analyzed concerted versus sequential PCET mechanisms in the context 
of exact quantum simulations, ${ }^{89}$ the RPMD simulations presented here constitute a trajectory-based simulation approach for the detailed, side-by-side comparison of concerted and sequential PCET mechanisms and thermal reaction rates, with both reaction mechanisms treated on a consistent dynamical footing.

\section{B. Reactions across multiple coupling regimes}

In this section, we employ RPMD simulations to investigate concerted PCET in a range of physical regimes, including the fully non-adiabatic, partially adiabatic, and fully adiabatic regimes. We validate the accuracy of the RPMD method by comparing thermal reaction rates obtained using the simulation method with those obtained using previously developed rate theories, and we investigate the variety of electron and proton tunneling processes that accompany concerted PCET. However, before delving into this analysis of PCET reactions, we first use RPMD to examine the crossover between electronically non-adiabatic (i.e., weak electronic coupling) and electronically adiabatic (i.e., strong electronic coupling) regimes for pure ET; analysis of this more simple process will provide useful context for the subsequent discussion of PCET.

\section{ET across electronic-coupling regimes}

Figure 5(a) presents the reaction rates for Systems $4 \mathrm{a}-4 \mathrm{~g}$, computed using RPMD (red), the electronically adiabatic ET rate expression (Eq. (15), blue), and the electronically nonadiabatic ET rate expression (Eq. (22), black). The results are plotted as a function of the temperature-reduced electronic coupling $\beta V_{\mathrm{ET}}$. For the weak-coupling regime $\left(\beta V_{\mathrm{ET}} \ll 1\right)$, the non-adiabatic rate expression constitutes the reference re- sult, whereas for the strong-coupling regime $\left(\beta V_{\mathrm{ET}} \gg 1\right)$, the adiabatic rate expression is the reference. It is clear that the RPMD rate correctly transitions between agreement with the non-adiabatic rate theory results at weak electronic coupling and the adiabatic rate theory results at strong electronic coupling. For systems with weak electronic coupling, we have shown previously that RPMD accurately describes the ET reaction rate throughout the normal and activationless regimes for the thermodynamic driving force, ${ }^{33}$ which follows from the method's exact description of statistical fluctuations ${ }^{24-26}$ and its formal connection to semiclassical instanton theory for deep-tunneling processes. ${ }^{24,90-93}$ Figure 5(a) shows that for symmetric systems, the accuracy of the method extends from the weak-coupling to the strong-coupling limits.

It is important to note that the RPMD rates in Fig. 5(a) are obtained without prior knowledge or assumption of the electronic coupling regime, and at no point in the RPMD rate calculation is $V_{\mathrm{ET}}$ required. A natural question, therefore, is whether a posteriori analysis of the trajectories from the RPMD rate calculation can be used to determine the electronic coupling regime for a given reaction. Figures 5(b)-5(d) demonstrate that this is indeed the case.

Figures 5(b) and 5(c) present snapshots of the electron position in a ring-polymer configuration at the reaction dividing surface, with the system either in the weak-coupling regime (b) or in the strong coupling regime (c). As described in Sec. V, the dividing surface used for the RPMD ET rate calculations is given by $f_{\mathrm{b}}=0$, which corresponds to configurations for which the electron position evenly spans the two redox sites and for which the solvent is depolarized to accommodate this symmetric charge distribution for the electron. ${ }^{33}$ At left in Figs. 5(b) and 5(c), we plot the electron position as a function of the ring-polymer bead index, $\alpha$, where $\tau=\beta \hbar \alpha / n_{\mathrm{e}}$. At right, we schematically illustrate the

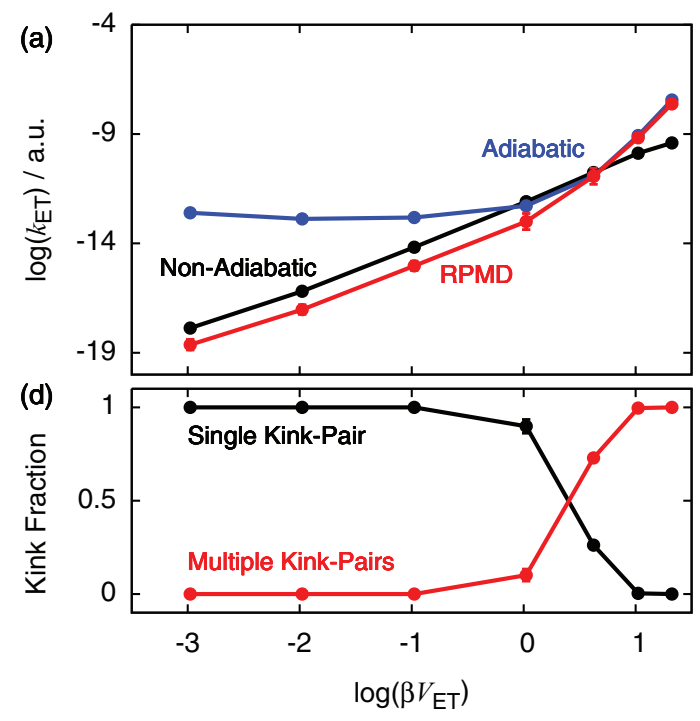

(b)
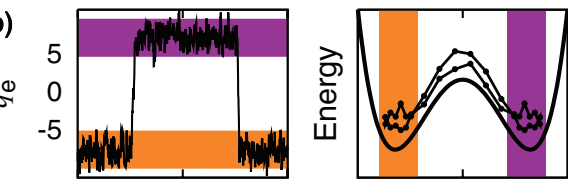

(c)
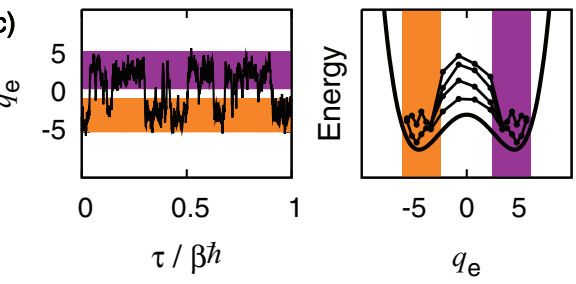

FIG. 5. (a) ET reaction rates as a function of the temperature-reduced electronic coupling, obtained using RPMD (red), the non-adiabatic rate expression for ET (Eq. (22), black), and the adiabatic rate expression for ET (Eq. (15), blue) for Systems 4a-4g. (b) and (c) At left, the electron position as a function of the ring-polymer bead index for (b) System $4 \mathrm{a}\left(\log \left(\beta V_{\mathrm{ET}}\right)=-2.98\right)$ and (c) System $4 \mathrm{~g}\left(\log \left(\beta V_{\mathrm{ET}}\right)=1.32\right)$; at right, a schematic illustration of the corresponding double-well potentials that are experienced by the transferring electron at the dividing surface, as well as the ring-polymer configurations in the electron position coordinate. The orange and purple stripes indicate the positions of the electron donor and acceptor sites, respectively. (d) The fraction of ring-polymer configurations at the dividing surface for ET that contain either a single kink-pair (black) or multiple kink-pairs (red) as a function of the temperature-reduced electronic coupling. 
double-well potential that is experienced by the transferring electron at the dividing surface, as well as the ringpolymer configuration in the electron position coordinate. Note that for the weak-coupling regime (Fig. 5(b)), the configuration exhibits only a single kink-pair, in which the electron position transits between the redox sites as a function of the ring-polymer bead index; for the strong-coupling regime (Fig. 5(c)), the configuration exhibits multiple kink-pairs.

It has long been recognized that the thermodynamic weight of ring-polymer kink-pair configurations is related to the eigenstate splitting (i.e., coupling) in symmetric doublewell systems. ${ }^{24,75,92-97}$ In particular, the weak-coupling regime corresponds to that for which the thermodynamic weight of ring-polymer configurations with multiple kinkpairs is small in comparison to the thermodynamic weight of ring-polymer configurations with only a single kink-pair; in the strong-coupling regime, configurations with multiple kink-pairs predominate. A straightforward approach to determining the coupling regime from the RPMD reactive trajectories is thus to simply count the fraction of ring-polymer configurations that exhibit multiple kink-pairs during the reactive transition event.

For Systems 4a-4g, Fig. 5(d) presents the results of this strategy, in which RPMD results are used for the a posteriori determination of the regime of the electronic coupling. For each system, we calculate the fraction of ring-polymer configurations that exhibit either a single kink-pair (black) or multiple kink-pairs (red) in the ensemble from which the RPMD trajectories are initialized in the rate calculation (i.e., the equilibrium ensemble constrained to the dividing surface). Here, a kink is defined as a segment of the ring-polymer for which the electron position spans from the donor region $\left(q_{\mathrm{e}}\right.$ $\left.<-0.7 \sigma_{\mathrm{e}}\right)$ to the acceptor region $\left(q_{\mathrm{e}}>0.7 \sigma_{\mathrm{e}}\right)$, where $\sigma_{\mathrm{e}}$ is the standard deviation of the ring-polymer bead position in the dividing surface ensemble. We note that more sophisticated strategies for identifying the ring-polymer configurations in the transition region may be needed for systems in which the trajectories exhibit extensive recrossing through a given dividing surface, ${ }^{58,84}$ although that is not the case for the systems considered here. It is immediately clear from the comparison of Figs. 5(a) and 5(d) that the onset of multiple kink-pair configurations coincides with the crossover between the adiabatic and non-adiabatic regimes for pure ET reactions at $\beta V_{\mathrm{ET}} \approx 1$.

We have thus shown that RPMD allows for the accurate calculation of the ET reaction rate across multiple regimes, without prior assumption of the electronic coupling regime, and it also enables determination of the coupling regime via simple analysis of the reactive trajectories.

\section{Concerted PCET across electronic-coupling regimes}

We now shift our attention to Systems 2a-2f, which exhibit weak vibrational coupling and which vary in electronic coupling from the weak- to strong-coupling regimes. Figure 6(a) presents the thermal reaction rate for concerted PCET in these systems, calculated using the fully nonadiabatic rate theory (Eq. (19), black), the partially adiabatic rate theory (Eq. (16), blue), and the RPMD method (red). For the weak-coupling regime $\left(\beta V_{\mathrm{ET}} \ll 1\right)$, the fully non-adiabatic rate expression constitutes the reference result, whereas for the strong-coupling regime $\left(\beta V_{\mathrm{ET}} \gg 1\right)$, the partially adiabatic rate expression is the reference; the fully nonadiabatic results are discontinued (open-circle) at values of the electronic coupling for which the diabatic-state localization procedure becomes ill defined (Sec. V B). As observed for the pure ET reactions, the RPMD method transitions correctly from the weak-coupling reference to the
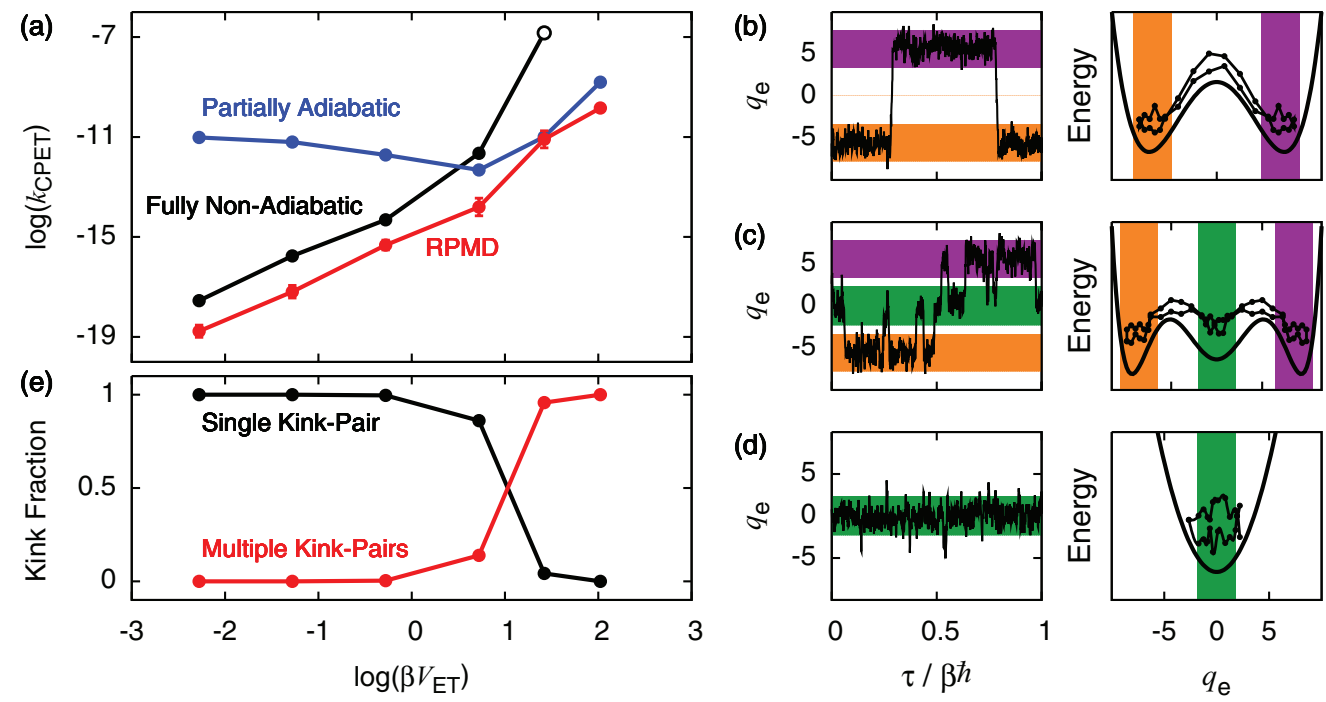

(c)
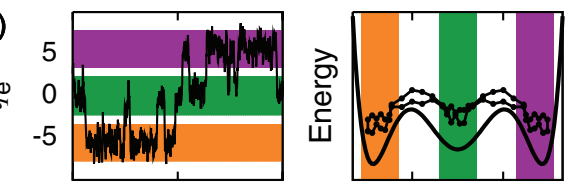

(d)
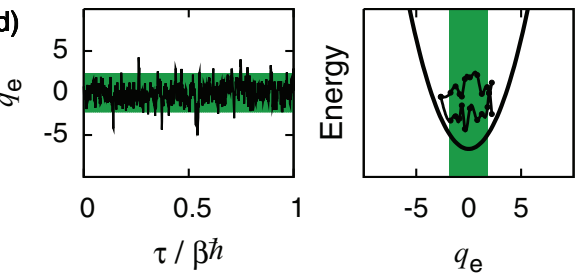

FIG. 6. (a) Concerted PCET reaction rates as a function of the temperature-reduced electronic coupling, obtained using RPMD (red), the fully non-adiabatic rate expression (Eq. (19), black) and the partially adiabatic rate expression (Eq. (16), blue) for Systems 2a-2f. (b)-(d) At left, the electron position as a function of the ring-polymer bead index for (b) System $2 \mathrm{a}\left(\log \left(\beta V_{\mathrm{ET}}\right)=-2.28\right)$, (c) System $2 \mathrm{~d}\left(\log \left(\beta V_{\mathrm{ET}}\right)=0.72\right)$, and $(\mathrm{d}) \operatorname{System} 2 \mathrm{f}\left(\log \left(\beta V_{\mathrm{ET}}\right)=2.02\right)$; at right, a schematic illustration of the corresponding potentials that are experienced by the transferring electron at the dividing surface, as well as the ring-polymer configurations in the electron position coordinate. The orange, purple, and green stripes indicate the positions of the electron donor site, the electron acceptor site, and transferring proton, respectively. (e) The fraction of ring-polymer configurations at the dividing surface for concerted PCET that contain either a single kink-pair (black) or multiple kink-pairs (red) as a function of the temperature-reduced electronic coupling. 
strong-coupling reference, while avoiding any assumptions about the coupling regime and while avoiding explicit calculation of the electronic or vibrational coupling.

As for the pure ET reactions, we can analyze the ensemble of reactive RPMD trajectories for concerted PCET to elucidate the associated tunneling processes and to determine the electronic coupling regime for each system. Figures 6(b)-6(d) present snapshots of a typical electron ring-polymer configuration at the concerted PCET reaction dividing surface, with the system either in the weak-coupling regime (b), the intermediate-coupling regime (c), or in the strong coupling regime (d). In each case, the dividing surface corresponds to configurations for which the electron and proton positions are distributed between the donor and acceptor sites; for such configurations the solvent is depolarized to accommodate this symmetric charge distribution. The left panel in Figs. 6(b)-6(d) presents the electron position as a function of the ring-polymer bead index; the right panels schematically illustrate the potential that is experienced by the transferring electron at the dividing surface, as well as the ring-polymer configurations in the electron position coordinate.

For the regime of weak electronic coupling (Fig. 6(b)), the electronic tunneling event that accompanies the PCET reaction is qualitatively similar to that observed for pure ET (Fig. 5(b)); the electron ring-polymer directly transitions between the two redox sites, exhibiting a single kink-pair. The coincident transfer of the proton in this regime simply affects the electron tunneling event by increasing the effective electronic coupling of the donor and acceptor redox sites, such that the concerted PCET mechanism may be described as proton-mediated electron superexchange. However, for the regime of strong electronic coupling (Fig. 6(d)), the electron transitions between the two redox sites via a mechanism that is fundamentally different than that observed for the pure ET reactions (Fig. 5(c)); in the PCET reaction, the electron collapses to a localized configuration about the position of the transferring proton, such that it adiabatically "rafts" with the proton between the donor and acceptor sites. This concerted PCET mechanism is immediately recognized as hydrogen atom transfer, or HAT. ${ }^{10,98-100}$

In both limiting regimes for the electronic coupling (Figs. 6(b) and 6(d)), the RPMD trajectories reveal concerted PCET reaction mechanisms that are implicit in the associated PCET rate theories (Eqs. (16) and (19)). However, the RPMD simulations additionally reveal a distinct - and to our knowledge, previously undiscussed - mechanism for concerted PCET in the intermediate coupling regime, in which the tunneling electron partially localizes about three sites: the positions of the electron donor site, the electron acceptor site, and the proton that is simultaneously undergoing transfer (Fig. 6(c)). This intermediate mechanism, which might be called "transient-proton-bridge" PCET, exhibits hybrid features of the PCET mechanisms from both limiting regimes (Figs. 6(b) and 6(d)), and it reflects the changing parameters that are employed to modulate the electronic coupling in Systems 2a-2f (Table VII); in this sense, it appears to be a physically reasonable mechanism for PCET in systems with intermediate electronic coupling, rather than an artifact of the approximate RPMD dynamics. Nonetheless, the transient- proton-bridge mechanism is certainly one for which no previous PCET rate theory has been derived, and it remains to be seen whether an unambiguous kinetic signature of this new mechanism can be identified and observed in a physical system.

Finally, Fig. 6(e) demonstrates that analysis of kink-pair formation in the reactive RPMD trajectories allows for the determination of the electronic coupling regime for the PCET reactions. As in Fig. 5(d), we present the calculated fraction of ring-polymer configurations that exhibit either a single kink-pair (black) or multiple kink-pairs (red) for the electron position in the equilibrium path-integral ensemble constrained to the dividing surface. We employ the same definition for a ring-polymer kink as in Sec. VI B 1, and we note that this definition registers multiple kink-pairs in the ringpolymer configurations in Figs. 6(c) and 6(d). Comparison of Figs. 6(a) and 6(e) demonstrates that the onset of multiple kink-pair configurations coincides with the crossover between the fully non-adiabatic and the partially adiabatic regimes for concerted PCET reactions.

\section{Concerted PCET across vibrational-coupling regimes}

We now analyze Systems 3a-3e, which exhibit strong electronic coupling and which vary in vibrational
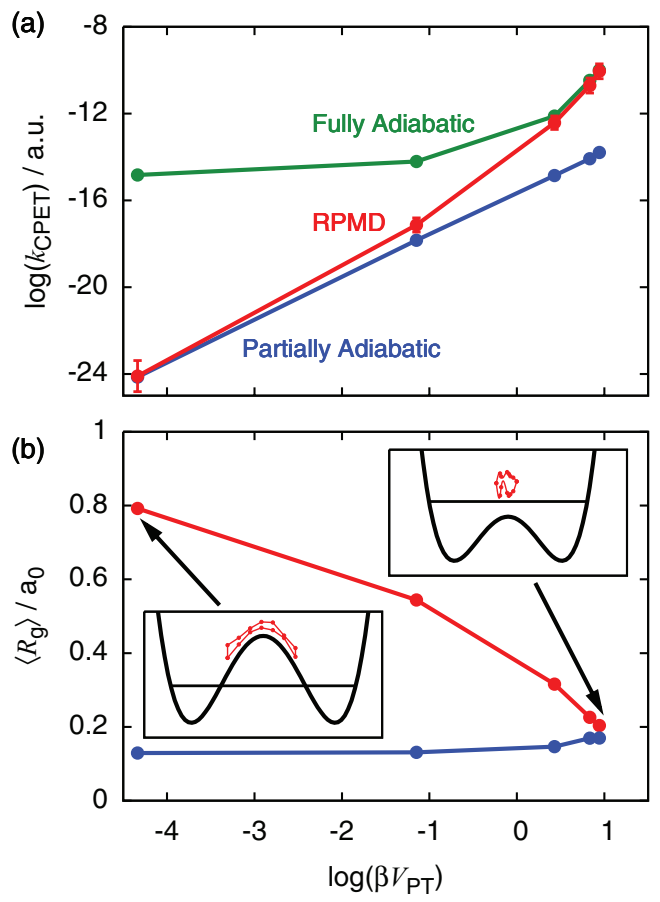

FIG. 7. (a) Concerted PCET reaction rates as a function of the temperaturereduced vibrational coupling, obtained using RPMD (red), the partially adiabatic rate expression (Eq. (16), blue), and the fully adiabatic rate expression (Eq. (15), green) for Systems 3a-3e. (b) The expectation value for the radius of gyration in the proton coordinate (Eq. (50)) calculated either in the ensemble for the PCET reactant basin (blue) or the ensemble constrained to the PCET dividing surface (red). The insets schematically illustrate the potential that is experienced by the transferring proton and the lowest vibrational eigenstate for the proton at the concerted PCET dividing surface, as well as the ring-polymer configurations in the proton position coordinate for System 3a $\left(\log \left(\beta V_{\mathrm{PT}}\right)=-4.3\right.$, bottom-left $)$ and System $3 \mathrm{e}\left(\log \left(\beta V_{\mathrm{PT}}\right)=0.95\right.$, top-right). 
coupling from the weak- to strong-coupling regimes. Figure 7(a) presents the thermal reaction rate for concerted PCET in these systems, calculated using the partially adiabatic rate theory (Eq. (16), blue), the fully adiabatic rate theory (Eq. (15), green), and RPMD (red). The results are plotted as a function of the temperature-reduced vibrational coupling $\beta V_{\mathrm{PT}}$. For the weak-coupling regime $\left(\beta V_{\mathrm{PT}} \ll 1\right)$, the partially adiabatic rate expression constitutes the reference result, whereas for the strong-coupling regime $\left(\beta V_{\mathrm{PT}} \gg 1\right)$, the fully adiabatic rate expression is the reference. The figure clearly shows that RPMD correctly transitions from the reference result in the regime of weak vibrational coupling to the reference for the strong-coupling regime.

In Secs. VI B 1 and VI B 2 we have demonstrated that analysis of the RPMD trajectories provides insight into the electron tunneling processes that accompany ET and concerted PCET; here, we show that the same is true for the proton tunneling events. Figure 7(b) presents the expectation value for the radius of gyration in the proton coordinate $(\mathrm{Eq}$. (50)) calculated either in the ensemble for the PCET reactant basin (blue) or the ensemble constrained to the PCET dividing surface (red). The radius of gyration in the proton coordinate is given by

$$
R_{\mathrm{g}}=\sqrt{\frac{1}{n_{\mathrm{p}}} \sum_{\gamma=1}^{n_{\mathrm{p}}}\left(q_{\mathrm{p}}^{(\gamma)}-\bar{q}_{\mathrm{p}}\right)^{2}} .
$$

For typical configurations in the dividing surface region, the insets in Fig. 7(b) schematically illustrate the potential that is experienced by the transferring proton and the lowest vibrational eigenstate for the proton, as well as the ringpolymer configurations in the proton position coordinate. For the weak-coupling regime, the ring polymer in the proton position "drapes" across the top of the potential barrier in the dividing surface configuration, leading to large values of $R_{\mathrm{g}}$ compared to the values in the reactant basin; for the strong-coupling regime, the proton exhibits more localized configurations. Using the connection between RPMD and semiclassical instanton theory, ${ }^{90,91}$ we recognize that a distended configuration of the ring polymer at the dividing surface indicates that the system is in the deep-tunneling regime. It is thus clear that the transition from strong vibrational coupling to weak vibrational coupling coincides with the onset of deep-tunneling for the proton coordinate. Comparison of Figs. 7(a) and 7(b) indicate that the onset of the deeptunneling regime tracks the transition of the RPMD rates between the results calculated using the fully adiabatic and partially adiabatic rate expressions.

\section{CONCLUSIONS}

In this work, we have extended the RPMD method to allow for the direct simulation of condensed-phase PCET reactions across a wide range of physically relevant regimes. The results presented here provide validation of the method for the description of PCET reactions, as well as a unifying mechanistic picture for PCET in different regimes of electronic and vibrational coupling.
We have demonstrated that the RPMD approach enables direct investigation of the competition between the concerted and sequential mechanisms for thermally activated PCET reactions, with both mechanisms treated on a consistent dynamical footing. For a system in the fully non-adiabatic regime, RPMD trajectories reveal distinct kinetic pathways associated with sequential and concerted PCET reaction mechanisms (Fig. 2); it was demonstrated that concerted PCET is favored in this system and that the concerted mechanism proceeds by a solvent-gating mechanism in which the reorganization energy is mitigated by charge cancellation among the transferring particles (Fig. 4). This analysis illustrates the potential usefulness of the RPMD method for the determination of PCET reaction mechanisms in systems that would otherwise be ambiguous on the basis of thermodynamic arguments alone, ${ }^{3}$ such as the PCET reaction of bis(imidazole) iron tetraphenylporphyrins linked to an ascorbate derivative. ${ }^{101}$

We have also validated the RPMD method for the PCET class of reactions by computing the thermal reaction rates for concerted PCET across a wide range of electronic and vibrational coupling regimes, including the fully nonadiabatic (electronically and vibrationally non-adiabatic), partially adiabatic (electronically adiabatic, but vibrationally non-adiabatic), and fully adiabatic (both electronically and vibrationally adiabatic) limits. Comparison of RPMD reaction rates with the results of previously derived PCET rate theories for each limiting regime demonstrates the accuracy of the direct simulation method in each case (Figs. 6(a) and 7(a)); it is particularly notable that RPMD correctly predicts the crossover in the thermal reaction rates between different coupling regimes, while avoiding the explicit calculation of the electronic or vibrational coupling and avoiding a priori assumptions about the coupling regime for the reaction. Moreover, by utilizing the connections between RPMD rate theory and semiclassical instanton theory, we showed that analysis of ring-polymer configurations in the RPMD transition path ensemble enables the a posteriori determination of the electronic and vibrational coupling regime for the reaction (Figs. 6(e) and 7(b)). These results indicate the potential utility of the RPMD method both for the calculation of PCET reaction rates in systems that span a broad range of physical regimes and for the determination of the electronic and vibrational coupling regime in systems for which these attributes are unknown.

Finally, we have used the RPMD method to identify and characterize a new PCET reaction mechanism that arises in the intermediate regime between fully nonadiabatic and partially adiabatic PCET (Fig. 6(c)). In the limiting regimes of electronic and vibrational coupling, analysis of the reactive RPMD trajectories reveals previously anticipated electron and proton tunneling processes that accompany concerted PCET reactions, including the proton-mediated electron superexchange mechanism for the fully nonadiabatic regime (Fig. 6(b)) and the HAT mechanism for the partially adiabatic regime (Fig. 6(d)). However, the RPMD simulations also reveal a distinct "transient-proton-bridge" mechanism in the crossover between these limiting regimes, in which the transferring electron partially localizes on the positions of the donor site, the acceptor site, and the proton 
that is simultaneously undergoing transfer (Fig. 6(c)). This progression of PCET mechanisms from superexchange to HAT as a function of electronic coupling suggests that concerted PCET may bear analogy to bridge-mediated ET reactions. ${ }^{71,102-104}$ Furthermore, the identification of a new mechanism in the RPMD simulations illustrates the capacity of the direct simulation approach to reveal unanticipated, yet physically reasonable, reaction mechanisms for condensedphase PCET.

\section{ACKNOWLEDGMENTS}

This work was supported by the National Science Foundation (NSF) CAREER Award under Grant No. CHE1057112 and the (U.S.) Department of Energy (DOE) under Grant No. DE-SC0006598. Additionally, J.S.K. acknowledges support from a NSF Graduate Research Fellowship under Grant No. DGE-1144469, and T.F.M. acknowledges support from a Camille and Henry Dreyfus Foundation New Faculty Award and an Alfred P. Sloan Foundation Research Fellowship. Computing resources were provided by the National Energy Research Scientific Computing Center (NERSC) (DE-AC02-05CH11231) and the Oak Ridge Leadership Computing Facility (OLCF) (DE-AC05-00OR22725). The authors sincerely thank Artur Menzeleev and Nandini Ananth for helpful conversations.

\section{APPENDIX A: COEFFICIENTS FOR THE ELECTRON POTENTIAL}

This appendix presents the coefficients associated with the quadratic functions in Eq. (26). The coefficients are provided in Table $\mathrm{V}$ and are chosen such that $U_{\mathrm{e}}\left(q_{\mathrm{e}}\right)$ is continuous and its derivative is piecewise continuous over the full range of $q_{\mathrm{e}}$.

TABLE V. Parameters for the donor coulombic well in the intrinsic electron potential energy function of Eq. (26). ${ }^{\mathrm{a}}$

\begin{tabular}{lccccc}
\hline \hline System & $a_{\mathrm{D}}$ & $b_{\mathrm{D}}$ & $c_{\mathrm{D}}$ & $r_{\mathrm{D}}^{\text {in }}$ & $r_{\mathrm{D}}^{\text {out }}$ \\
\hline 1 & 0.2266300 & 2.6933600 & 4.9465600 & -4.0000 & -8.0000 \\
$\mathrm{2a}$ & 0.2266300 & 2.6933600 & 4.9465600 & -4.0000 & -8.0000 \\
$\mathrm{2b}$ & 0.1824656 & 2.1684926 & 3.9826011 & -4.0000 & -8.0000 \\
$\mathrm{2c}$ & 0.1523821 & 1.8109689 & 3.3259817 & -4.0000 & -8.0000 \\
$2 \mathrm{~d}$ & 0.1379922 & 1.6399537 & 3.0118994 & -4.0000 & -8.0000 \\
$\mathrm{2e}$ & 0.1298241 & 1.5428811 & 2.8336183 & -4.0000 & -8.0000 \\
$2 \mathrm{f}$ & 0.1067039 & 1.2681114 & 2.3289829 & -4.0000 & -8.0000 \\
$3 \mathrm{a}-3 \mathrm{e}$ & 0.0743542 & 0.5740833 & 0.0207500 & -2.0000 & -6.0000 \\
$4 \mathrm{a}$ & 0.0932266 & 1.3904813 & 3.9598592 & -5.4940 & -9.4940 \\
$4 \mathrm{~b}$ & 0.0929362 & 1.2049293 & 2.6659598 & -4.5310 & -8.5310 \\
$4 \mathrm{c}$ & 0.0923649 & 1.0121182 & 1.5130165 & -3.5471 & -7.5471 \\
$4 \mathrm{~d}$ & 0.0910333 & 0.8059524 & 0.4947511 & -2.5321 & -6.5321 \\
$4 \mathrm{e}$ & 0.0891837 & 0.6684716 & -0.0621548 & -1.8960 & -5.8960 \\
$4 \mathrm{f}$ & 0.0867814 & 0.5657624 & -0.4151582 & -1.4570 & -5.4570 \\
$4 \mathrm{~g}$ & 0.0834663 & 0.4753245 & -0.6820558 & -1.1070 & -5.1070 \\
\hline \hline
\end{tabular}

${ }^{\text {a The parameters for the acceptor coulombic well are given by } a_{\mathrm{A}}=a_{\mathrm{D}}, b_{\mathrm{A}}=-b_{\mathrm{D}}, c_{\mathrm{A}}}$ $=c_{\mathrm{D}}, r_{\mathrm{A}}^{\text {in }}=-r_{\mathrm{D}}^{\text {in }}$, and $r_{\mathrm{A}}^{\text {out }}=-r_{\mathrm{D}}^{\text {out }}$. All parameters are given in atomic units.
TABLE VI. Parameters for the PCET and ET systems. ${ }^{a}$

\begin{tabular}{lc}
\hline \hline Parameter & \\
\hline$f$ & 12 \\
$\omega_{\mathrm{s}}$ & $2.3221 \times 10^{-4}$ \\
$\omega_{\mathrm{c}}$ & $2.3221 \times 10^{-4}$ \\
$M$ & 25539 \\
$m_{\mathrm{s}}$ & 25539 \\
$m_{\mathrm{e}}$ & 1.0 \\
$m_{\mathrm{p}}$ & 1836.1 \\
$\eta / M \omega_{\mathrm{c}}$ & 1.0 \\
\hline \hline
\end{tabular}

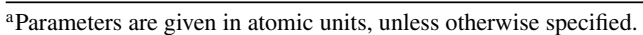

\section{APPENDIX B: SYSTEM-BATH POTENTIAL ENERGY PARAMETERS}

This appendix describes parameters for the system-bath models for condensed-phase PCET and ET. Table VI presents parameters that are common to all PCET and ET model systems; the cutoff and solvent frequencies, $\omega_{\mathrm{c}}$ and $\omega_{\mathrm{s}}$, are obtained from the spectral density of acetonitrile, ${ }^{105}$ the masses of the solvent mode and bath oscillators, $m_{s}$ and $M$, correspond to that of a nitrogen atom, and the friction coefficient, $\eta$, is chosen to be consistent with a condensed-phase environment. ${ }^{33,77}$ Table VII presents potential energy function parameters for System 1, Systems 2a-2f, and Systems 3a-3e. Table VIII presents potential energy function parameters for Systems $4 \mathrm{a}-4 \mathrm{~g}$.

\section{APPENDIX C: AUXILIARY RESTRAINING POTENTIALS}

This appendix describes auxiliary restraining potentials that are introduced for the PIMD sampling trajectories used in the calculation of 1D FE profiles and in the initial sampling of configurations for the RPMD trajectories. These auxiliary restraining potentials simply prevent the PIMD sampling trajectories from visiting configurations outside of the sequential or concerted PCET channel of interest.

TABLE VII. Potential energy function parameters for the PCET systems. ${ }^{\text {a }}$

\begin{tabular}{lcccccccc}
\hline \hline System & $r_{\mathrm{D}}$ & $r_{\mathrm{A}}$ & $\mu_{\mathrm{e}}$ & $\omega_{\mathrm{p}}$ & $V_{0}$ & $\mu_{\mathrm{es}}$ & $\mu_{\mathrm{ps}}$ & $\mu_{\mathrm{ep}}$ \\
\hline 1 & -6.0 & 6.0 & 3.6680 & 0.0131 & 23.7 & -1.20 & 6.00 & 1.3 \\
$2 \mathrm{a}$ & -6.0 & 6.0 & 3.6680 & 0.011 & 20.0 & -1.00 & 4.95 & 1.3 \\
$\mathrm{2b}$ & -6.0 & 6.0 & 2.9532 & 0.011 & 20.0 & -1.01 & 4.95 & 1.3 \\
$\mathrm{2c}$ & -6.0 & 6.0 & 2.4663 & 0.011 & 20.0 & -1.06 & 4.95 & 1.3 \\
$\mathrm{2d}$ & -6.0 & 6.0 & 2.2334 & 0.011 & 20.0 & -1.25 & 4.95 & 1.3 \\
$\mathrm{2e}$ & -6.0 & 6.0 & 2.1012 & 0.011 & 20.0 & -2.01 & 4.95 & 1.3 \\
$2 \mathrm{f}$ & -6.0 & 6.0 & 1.7270 & 0.011 & 20.0 & -3.30 & 1.00 & 1.3 \\
$3 \mathrm{a}$ & -4.0 & 4.0 & 1.245 & 0.009 & 20.0 & -1.55 & 0.0 & 0.15 \\
$3 \mathrm{~b}$ & -4.0 & 4.0 & 1.245 & 0.009 & 10.0 & -1.51 & 0.0 & 0.15 \\
$3 \mathrm{c}$ & -4.0 & 4.0 & 1.245 & 0.009 & 5.0 & -1.43 & 0.0 & 0.20 \\
$3 \mathrm{~d}$ & -4.0 & 4.0 & 1.245 & 0.009 & 3.0 & -1.40 & 0.0 & 0.30 \\
$3 \mathrm{e}$ & -4.0 & 4.0 & 1.245 & 0.009 & 2.3 & -1.40 & 0.0 & 0.35 \\
\hline \hline
\end{tabular}

${ }^{\mathrm{a}} V_{0}, \mu_{\mathrm{es}}$, and $\mu_{\mathrm{ps}}$ are given in units of a.u. $\times 10^{-3}$; all other parameters are given in atomic units. 
TABLE VIII. Potential energy function parameters for the ET systems. ${ }^{\text {a }}$

\begin{tabular}{lcccc}
\hline \hline System & $r_{\mathrm{D}}$ & $r_{\mathrm{A}}$ & $\mu_{\mathrm{e}}$ & $\mu_{\mathrm{es}}$ \\
\hline $4 \mathrm{a}$ & -7.4940 & 7.4940 & 1.5 & -0.94 \\
$4 \mathrm{~b}$ & -6.5310 & 6.5310 & 1.5 & -1.10 \\
$4 \mathrm{c}$ & -5.5471 & 5.5471 & 1.5 & -1.30 \\
$4 \mathrm{~d}$ & -4.5321 & 4.5321 & 1.5 & -1.60 \\
$4 \mathrm{e}$ & -3.8960 & 3.8960 & 1.5 & -1.89 \\
$4 \mathrm{f}$ & -3.4570 & 3.4570 & 1.5 & -2.24 \\
$4 \mathrm{~g}$ & -3.1070 & 3.1070 & 1.5 & -2.80 \\
\hline \hline
\end{tabular}

${ }^{\mathrm{a}} \mu_{\mathrm{es}}$ is given in units of a.u. $\times 10^{-3}$; all other parameters are given in atomic units.

\section{Auxiliary restraining potential for concerted PCET}

For Systems 1 and 2a-2e, which exhibit distinct channels for sequential and concerted PCET reactions, we now discuss the auxiliary restraining potential introduced to restrict equilibrium sampling of the system to the concerted channel. This potential is given by

$$
U_{\mathrm{aux}}\left(\bar{q}_{\mathrm{p}}, f_{\mathrm{b}}\right)= \begin{cases}a_{\mathrm{aux}}\left[\bar{q}_{\mathrm{p}}-q_{+}\left(f_{\mathrm{b}}\right)\right]^{2}, & \bar{q}_{\mathrm{p}}>q_{+}\left(f_{\mathrm{b}}\right) \\ a_{\mathrm{aux}}\left[\bar{q}_{\mathrm{p}}-q_{-}\left(f_{\mathrm{b}}\right)\right]^{2}, & \bar{q}_{\mathrm{p}}<q_{-}\left(f_{\mathrm{b}}\right), \\ 0, & \text { otherwise }\end{cases}
$$

where

$$
q_{+}\left(f_{\mathrm{b}}\right)=b_{\mathrm{aux}} f_{\mathrm{b}}+c_{\mathrm{aux}}
$$

and

$$
q_{-}\left(f_{\mathrm{b}}\right)=b_{\mathrm{aux}} f_{\mathrm{b}}-c_{\mathrm{aux}} .
$$

The coefficients $a_{\mathrm{aux}}, b_{\mathrm{aux}}$, and $c_{\mathrm{aux}}$ (Table IX) are chosen to restrict the system to the concerted channel.

\section{Auxiliary restraining potential for ET prior to PT}

For System 1, we now discuss the auxiliary restraining potential introduced to restrict equilibrium sampling of the system to the ET channel connecting the OU and RU species in the sequential mechanism. This potential is given by

$$
U_{\mathrm{SET}}\left(\bar{q}_{\mathrm{p}}\right)=\left\{\begin{array}{ll}
a_{\mathrm{SET}}\left(\bar{q}_{\mathrm{p}}-b_{\mathrm{SET}}\right)^{2}, & \bar{q}_{\mathrm{p}}<b_{\mathrm{SET}} \\
0, & \text { otherwise }
\end{array} .\right.
$$

TABLE IX. Parameters for the auxiliary restraining potential in Eqs. (C1)-(C3). ${ }^{\text {a }}$

\begin{tabular}{lccc}
\hline \hline System & $a_{\text {aux }}$ & $b_{\text {aux }}$ & $c_{\text {aux }}$ \\
\hline 1 & 1.0 & 0.57 & 0.36 \\
$2 \mathrm{a}$ & 1.0 & 0.66 & 0.39 \\
$2 \mathrm{~b}$ & 1.0 & 0.67 & 0.40 \\
$2 \mathrm{c}$ & 1.0 & 0.69 & 0.41 \\
$2 \mathrm{~d}$ & 1.0 & 0.78 & 0.41 \\
$2 \mathrm{e}$ & 1.0 & 1.11 & 0.38 \\
\hline \hline
\end{tabular}

\begin{tabular}{|c|c|c|c|}
\hline System & $c_{\mathrm{ad}}^{(6)}$ & $c_{\mathrm{ad}}^{(5)}$ & $c_{\mathrm{ad}}^{(4)}$ \\
\hline $2 \mathrm{a}$ & $2.340 \times 10^{-5}$ & $-4.901 \times 10^{-4}$ & $1.545 \times 10^{-1}$ \\
\hline $2 b$ & $3.054 \times 10^{-7}$ & $-4.783 \times 10^{-4}$ & $1.538 \times 10^{-1}$ \\
\hline $2 \mathrm{c}$ & $1.776 \times 10^{-5}$ & $1.565 \times 10^{-3}$ & $1.452 \times 10^{-1}$ \\
\hline $2 d$ & $8.203 \times 10^{-6}$ & $1.441 \times 10^{-2}$ & $9.150 \times 10^{-2}$ \\
\hline $2 \mathrm{e}$ & $1.886 \times 10^{-3}$ & $-1.029 \times 10^{-2}$ & $1.737 \times 10^{-1}$ \\
\hline $2 f$ & $4.547 \times 10^{-5}$ & $5.369 \times 10^{-4}$ & $1.508 \times 10^{-1}$ \\
\hline $3 a$ & $1.122 \times 10^{-5}$ & $4.872 \times 10^{-3}$ & $4.441 \times 10^{-2}$ \\
\hline $3 b$ & $9.645 \times 10^{-6}$ & $4.111 \times 10^{-3}$ & $1.175 \times 10^{-1}$ \\
\hline $3 \mathrm{c}$ & $1.987 \times 10^{-5}$ & $2.061 \times 10^{-3}$ & $2.657 \times 10^{-1}$ \\
\hline $3 \mathrm{~d}$ & $2.553 \times 10^{-4}$ & $-8.242 \times 10^{-5}$ & $4.571 \times 10^{-1}$ \\
\hline $3 e$ & $2.064 \times 10^{-4}$ & $1.192 \times 10^{-4}$ & $5.970 \times 10^{-1}$ \\
\hline System & $c_{\mathrm{ad}}^{(3)}$ & $c_{\mathrm{ad}}^{(2)}$ & $c_{\mathrm{ad}}^{(1)}$ \\
\hline $2 a$ & $-2.182 \times 10^{-3}$ & $-1.191 \times 10^{-1}$ & $-4.128 \times 10^{-2}$ \\
\hline $2 b$ & $-1.910 \times 10^{-3}$ & $-1.204 \times 10^{-1}$ & $-4.248 \times 10^{-2}$ \\
\hline $2 c$ & $7.850 \times 10^{-3}$ & $-1.292 \times 10^{-1}$ & $-4.301 \times 10^{-2}$ \\
\hline $2 d$ & $9.529 \times 10^{-2}$ & $-2.042 \times 10^{-1}$ & $-2.031 \times 10^{-2}$ \\
\hline $2 \mathrm{e}$ & $-1.197 \times 10^{-2}$ & $-1.607 \times 10^{-1}$ & $-2.762 \times 10^{-4}$ \\
\hline $2 f$ & $1.201 \times 10^{-3}$ & $-1.373 \times 10^{-1}$ & $4.793 \times 10^{-5}$ \\
\hline $3 a$ & $5.452 \times 10^{-2}$ & $-1.493 \times 10^{-1}$ & $8.250 \times 10^{-4}$ \\
\hline $3 b$ & $4.522 \times 10^{-2}$ & $-1.369 \times 10^{-1}$ & $5.791 \times 10^{-4}$ \\
\hline $3 \mathrm{c}$ & $2.438 \times 10^{-2}$ & $-1.113 \times 10^{-1}$ & $4.775 \times 10^{-4}$ \\
\hline $3 d$ & $1.226 \times 10^{-2}$ & $-9.804 \times 10^{-2}$ & $3.088 \times 10^{-4}$ \\
\hline $3 e$ & $1.251 \times 10^{-2}$ & $-9.812 \times 10^{-2}$ & $3.207 \times 10^{-4}$ \\
\hline System & $c_{\mathrm{ad}}^{(0)}$ & & \\
\hline $2 \mathrm{a}$ & -2.948 & & \\
\hline $2 b$ & -2.389 & & \\
\hline $2 \mathrm{c}$ & -2.013 & & \\
\hline $2 \mathrm{~d}$ & -1.842 & & \\
\hline $2 \mathrm{e}$ & -1.782 & & \\
\hline $2 \mathrm{f}$ & -1.650 & & \\
\hline $3 a$ & -1.037 & & \\
\hline $3 b$ & -1.015 & & \\
\hline $3 c$ & $-9.758 \times 10^{-1}$ & & \\
\hline $3 d$ & $-9.576 \times 10^{-1}$ & & \\
\hline $3 \mathrm{e}$ & $-9.576 \times 10^{-1}$ & & \\
\hline
\end{tabular}

${ }^{\mathrm{a}} a_{\text {aux }}$ is given in units of a.u. $\times 10^{-2}$; all other parameters are given in atomic units.
TABLE X. Parameters for the polynomial fit to the lowest adiabatic electronic state (Eq. (42)). ${ }^{\mathrm{a}}$

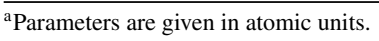

The coefficients $a_{\mathrm{SET}}=1.0 \times 10^{2}$ a.u. and $b_{\mathrm{SET}}=-0.21 a_{0}$ are chosen to correctly restrict the system to the ET channel.

\section{APPENDIX D: PARAMETERS FOR FITTING FUNCTIONS}

This appendix presents the coefficients associated with the polynomials fit to the proton potential energy surfaces in Eq. (42). The coefficients are provided in Table X.

\section{APPENDIX E: DIABATIZATION PROTOCOL}

This appendix describes the procedure for transforming the potential energy function from a position basis for the electron (Eq. (24)) to a diabatic basis in which the reactant and product states are maximally localized on the position of the electron donor and acceptor, respectively. The diabatization protocol presented below is similar to that used for ET reactions in Ref. 33. 
We begin by calculating the two lowest adiabatic electronic eigenstates $\left(\psi_{0}\left(q_{\mathrm{e}} ; q_{\mathrm{p}}, q_{\mathrm{s}}\right)\right.$ and $\left.\psi_{1}\left(q_{\mathrm{e}} ; q_{\mathrm{p}}, q_{\mathrm{s}}\right)\right)$ and eigenenergies $\left(\varepsilon_{0}\left(q_{\mathrm{p}}, q_{\mathrm{s}}\right)\right.$ and $\left.\varepsilon_{1}\left(q_{\mathrm{p}}, q_{\mathrm{s}}\right)\right)$ of the system Hamiltonian associated with $U_{\mathrm{sys}}\left(q_{\mathrm{e}}, q_{\mathrm{p}}, q_{\mathrm{s}}\right)$ (Eq. (25)). The 1D eigenvalue problem is solved at fixed values of the proton coordinate in the range $-1.5 a_{0} \leq q_{\mathrm{p}} \leq 1.5 a_{0}$ and with $q_{\mathrm{s}}=0$. For each value of $q_{\mathrm{p}}$ the system Hamiltonian is diagonalized on a uniform 1D DVR grid of 2048 electron positions in the range $-30 a_{0} \leq q_{\mathrm{e}} \leq 30 a_{0}$.

For each value of $q_{\mathrm{p}}$, reactant and product electronic wavefunctions in the diabatic basis are obtained via rotation of the two lowest energy adiabatic wavefunctions, using

$$
\begin{aligned}
\phi_{\mathrm{R}}\left(q_{\mathrm{e}} ; q_{\mathrm{p}}, q_{\mathrm{s}}\right)= & \cos \left(\theta_{\mathrm{p}}\right) \psi_{0}\left(q_{\mathrm{e}} ; q_{\mathrm{p}}, q_{\mathrm{s}}\right) \\
& -\sin \left(\theta_{\mathrm{p}}\right) \psi_{1}\left(q_{\mathrm{e}} ; q_{\mathrm{p}}, q_{\mathrm{s}}\right)
\end{aligned}
$$

and

$$
\begin{aligned}
\phi_{\mathrm{P}}\left(q_{\mathrm{e}} ; q_{\mathrm{p}}, q_{\mathrm{s}}\right)= & \sin \left(\theta_{\mathrm{p}}\right) \psi_{0}\left(q_{\mathrm{e}} ; q_{\mathrm{p}}, q_{\mathrm{s}}\right) \\
& +\cos \left(\theta_{\mathrm{p}}\right) \psi_{1}\left(q_{\mathrm{e}} ; q_{\mathrm{p}}, q_{\mathrm{s}}\right),
\end{aligned}
$$

where

$$
\theta_{\mathrm{p}}=\frac{1}{2} \arctan \left(\frac{S_{10}+S_{01}}{S_{11}-S_{00}}\right)
$$

and

$$
S_{u v}=\int_{-\infty}^{0} \psi_{u}\left(q_{\mathrm{e}} ; q_{\mathrm{p}}, q_{\mathrm{s}}\right)^{*} \psi_{v}\left(q_{\mathrm{e}} ; q_{\mathrm{p}}, q_{\mathrm{s}}\right) d q_{\mathrm{e}}
$$

This choice of the rotation angle, $\theta_{\mathrm{p}}$, maximizes $\int_{-\infty}^{0}\left|\phi_{\mathrm{R}}\left(q_{\mathrm{e}} ; q_{\mathrm{p}}, q_{\mathrm{s}}\right)\right|^{2} d q_{\mathrm{e}}$, the probability that the reactant diabatic state is positioned on the electron donor. Maximization of the probability that the product state is positioned on the electron acceptor yields an identical choice for $\theta_{\mathrm{p}}$. by

The corresponding reactant electronic diabat is thus given

$$
V_{\mathrm{R}}\left(q_{\mathrm{p}}, q_{\mathrm{s}}\right)=\varepsilon_{0}\left(q_{\mathrm{p}}, q_{\mathrm{s}}\right) \cos ^{2} \theta_{\mathrm{p}}+\varepsilon_{1}\left(q_{\mathrm{p}}, q_{\mathrm{s}}\right) \sin ^{2} \theta_{\mathrm{p}},
$$

and the product electronic diabat is given by

$$
V_{\mathrm{P}}\left(q_{\mathrm{p}}, q_{\mathrm{s}}\right)=\varepsilon_{0}\left(q_{\mathrm{p}}, q_{\mathrm{s}}\right) \sin ^{2} \theta_{\mathrm{p}}+\varepsilon_{1}\left(q_{\mathrm{p}}, q_{\mathrm{s}}\right) \cos ^{2} \theta_{\mathrm{p}} .
$$

The electronic coupling is given by

$$
V_{E T}\left(q_{\mathrm{p}}, q_{\mathrm{s}}\right)=\left(\varepsilon_{0}\left(q_{\mathrm{p}}, q_{\mathrm{s}}\right)-\varepsilon_{1}\left(q_{\mathrm{p}}, q_{\mathrm{s}}\right)\right) \cos \theta_{\mathrm{p}} \sin \theta_{\mathrm{p}} .
$$

${ }^{1}$ S. Hammes-Schiffer and A. A. Stuchebrukhov, Chem. Rev. 110, 6939 (2010).

${ }^{2}$ S. Y. Reece and D. G. Nocera, Annu. Rev. Biochem. 78, 673 (2009).

${ }^{3}$ J. J. Warren, T. A. Tronic, and J. M. Mayer, Chem. Rev. 110, 6961 (2010).

${ }^{4}$ J. L. Dempsey, J. R. Winkler, and H. B. Gray, Chem. Rev. 110, 7024 (2010).

${ }^{5}$ M. Y. Okamura, M. L. Paddock, M. S. Graige, and G. Feher, Biochim. Biophys. Acta Bioenerg. 1458, 148 (2000)

${ }^{6}$ M. Saraste, Science 283, 1488 (1999).

${ }^{7}$ M. Wikstrom, Curr. Opin. Struct. Biol. 8, 480 (1998).

${ }^{8}$ H. Michel, Biochemistry 38, 15129 (1999).

${ }^{9}$ S. Y. Reece, J. M. Hodgkiss, J. Stubbe, and D. G. Nocera, Philos. Trans. R. Soc. London, Ser. B 361, 1351 (2006).

${ }^{10}$ R. I. Cukier, J. Phys. Chem. B 106, 1746 (2002).

${ }^{11} \mathrm{~J}$. Ulstrup, Charge Transfer Processes in Condensed Media (SpringerVerlag, Berlin, 1979).
${ }^{12}$ Y. Georgievskii and A. A. Stuchebrukhov, J. Chem. Phys. 113, 10438 (2000).

${ }^{13}$ S. Hammes-Schiffer, Energy Environ. Sci. 5, 7696 (2012).

${ }^{14}$ R. I. Cukier and D. G. Nocera, Annu. Rev. Phys. Chem. 49, 337 (1998).

${ }^{15}$ M. H. V. Huynh and T. J. Meyer, Chem. Rev. 107, 5004 (2007).

${ }^{16}$ J. M. Mayer and I. J. Rhile, Biochim. Biophys. Acta Bioenerg. 1655, 51 (2004).

${ }^{17}$ R. I. Cukier, J. Phys. Chem. 98, 2377 (1994).

${ }^{18}$ R. I. Cukier, J. Phys. Chem. 100, 15428 (1996).

${ }^{19}$ A. Soudackov and S. Hammes-Schiffer, J. Chem. Phys. 113, 2385 (2000).

${ }^{20}$ A. Staib, D. Borgis, and J. T. Hynes, J. Chem. Phys. 102, 2487 (1995).

${ }^{21}$ D. Borgis and J. T. Hynes, J. Phys. Chem. 100, 1118 (1996).

${ }^{22}$ I. R. Craig and D. E. Manolopoulos, J. Chem. Phys. 121, 3368 (2004).

${ }^{23}$ R. P. Feynman and A. R. Hibbs, Quantum Mechanics and Path Integrals, International Series in Pure and Applied Physics (McGraw-Hill, New York, 1965)

${ }^{24}$ D. Chandler and P. G. Wolynes, J. Chem. Phys. 74, 4078 (1981).

${ }^{25}$ M. Parrinello and A. Rahman, J. Chem. Phys. 80, 860 (1984).

${ }^{26}$ B. D. Raedt, M. Sprik, and M. L. Klein, J. Chem. Phys. 80, 5719 (1984).

${ }^{27}$ R. Collepardo-Guevara, Y. V. Suleimanov, and D. E. Manolopoulos, J. Chem. Phys. 130, 174713 (2009).

${ }^{28}$ N. Boekelheide, R. Salomón-Ferrer, and T. F. Miller III, Proc. Natl. Acad. Sci. U.S.A. 108, 16159 (2011).

${ }^{29}$ R. Collepardo-Guevara, I. R. Craig, and D. E. Manolopoulos, J. Chem. Phys. 128, 144502 (2008).

${ }^{30}$ Y. V. Suleimanov, R. Collepardo-Guevara, and D. E. Manolopoulos, J. Chem. Phys. 134, 044131 (2011).

${ }^{31}$ T. F. Miller III, J. Chem. Phys. 129, 194502 (2008).

${ }^{32}$ A. R. Menzeleev and T. F. Miller III, J. Chem. Phys. 132, 034106 (2010).

${ }^{33}$ A. R. Menzeleev, N. Ananth, and T. F. Miller III, J. Chem. Phys. 135, 074106 (2011).

${ }^{34}$ T. F. Miller III and D. E. Manolopoulos, J. Chem. Phys. 123, 154504 (2005).

${ }^{35}$ T. F. Miller III and D. E. Manolopoulos, J. Chem. Phys. 122, 184503 (2005).

${ }^{36}$ S. Habershon, T. E. Markland, and D. E. Manolopoulos, J. Chem. Phys. 131, 024501 (2009)

${ }^{37}$ I. R. Craig and D. E. Manolopoulos, Chem. Phys. 322, 236 (2006).

${ }^{38}$ T. E. Markland, S. Habershon, and D. E. Manolopoulos, J. Chem. Phys. 128, 194506 (2008).

${ }^{39}$ Y. V. Suleimanov, J. Phys. Chem. C 116, 11141 (2012).

${ }^{40}$ T. E. Markland, J. A. Morrone, K. Miyazaki, B. J. Berne, D. R. Reichman, and E. Rabani, J. Chem. Phys. 136, 074511 (2012).

${ }^{41}$ P. Ehrenfest, Z. Phys. 45, 455 (1927).

${ }^{42}$ A. D. Mclachlan, Mol. Phys. 8, 39 (1964).

${ }^{43}$ J. C. Tully, J. Chem. Phys. 93, 1061 (1990).

${ }^{44}$ J. C. Tully, Faraday Discuss. 110, 407 (1998).

${ }^{45}$ J.-Y. Fang and S. Hammes-Schiffer, J. Chem. Phys. 106, 8442 (1997).

${ }^{46}$ A. Hazra, A. V. Soudackov, and S. Hammes-Schiffer, J. Phys. Chem. B 114, 12319 (2010).

${ }^{47}$ A. V. Soudackov, A. Hazra, and S. Hammes-Schiffer, J. Chem. Phys. 135, 144115 (2011).

${ }^{48}$ B. Auer, A. V. Soudackov, and S. Hammes-Schiffer, J. Phys. Chem. B 116, 7695 (2012).

${ }^{49}$ M. Ben-Nun, J. Quenneville, and T. J. Martinez, J. Phys. Chem. A 104, 5161 (2000).

${ }^{50}$ H. D. Meyer and W. H. Miller, J. Chem. Phys. 70, 3214 (1979).

${ }^{51}$ H. D. Meyer and W. H. Miller, J. Chem. Phys. 72, 2272 (1980).

${ }^{52}$ G. Stock and M. Thoss, Phys. Rev. Lett. 78, 578 (1997).

${ }^{53}$ P. Shuskhov, R. Li, and J. C. Tully, J. Chem. Phys. 137, 22 A549 (2012).

${ }^{54}$ S. Nielsen, R. Kapral, and G. Ciccotti, J. Chem. Phys. 115, 5805 (2001).

${ }^{55}$ J. R. Schmidt, P. V. Parandekar, and J. C. Tully, J. Chem. Phys. 129, 044104 (2008)

${ }^{56}$ I. R. Craig and D. E. Manolopoulos, J. Chem. Phys. 122, 084106 (2005).

${ }^{57}$ I. R. Craig and D. E. Manolopoulos, J. Chem. Phys. 123, 034102 (2005).

${ }^{58}$ P. G. Bolhuis, D. Chandler, C. Dellago, and P. L. Geissler, Annu. Rev. Phys. Chem. 53, 291 (2002).

${ }^{59}$ L. Maragliano, A. Fischer, E. Vanden-Eijnden, and G. Ciccotti, J. Chem. Phys. 125, 024106 (2006).

${ }^{60}$ R. P. Steele, J. Zwickl, P. Shushkov, and J. C. Tully, J. Chem. Phys. 134, 074112 (2011).

${ }^{61}$ E. Wigner, Z. Phys. Chem. Abt. B 19, 203 (1932).

${ }^{62}$ H. Eyring, J. Chem. Phys. 3, 107 (1935).

${ }^{63}$ J. C. Keck, J. Chem. Phys. 32, 1035 (1960). 
${ }^{64}$ W. H. Miller, J. Chem. Phys. 58, 1664 (1973).

${ }^{65}$ D. Chandler, J. Chem. Phys. 68, 2959 (1978).

${ }^{66}$ C. H. Bennet, in Algorithms for Chemical Computations, edited by R. E. Christofferson (American Chemical Society, Washington, DC, 1977), p. 63.

${ }^{67}$ E. A. Carter, G. Ciccotti, J. T. Hynes, and R. Kapral, Chem. Phys. Lett. 156, 472 (1989).

${ }^{68}$ G. K. Schenter, B. C. Garrett, and D. G. Truhlar, J. Chem. Phys. 119, 5828 (2003).

${ }^{69}$ J. B. Watney, A. V. Soudackov, K. F. Wong, and S. Hammes-Schiffer, Chem. Phys. Lett. 418, 268 (2006).

${ }^{70}$ R. A. Marcus, Annu. Rev. Phys. Chem. 115, 155 (1964).

${ }^{71}$ A. Nitzan, Chemical Dynamics in Condensed Phases (Oxford University Press, 2006).

${ }^{72}$ R. A. Marcus, Discuss. Faraday Soc. 29, 21 (1960).

${ }^{73}$ R. A. Marcus, J. Chem. Phys. 43, 3477 (1965).

${ }^{74}$ R. A. Marcus and N. Sutin, Biochim. Biophys. Acta 811, 265 (1985).

${ }^{75}$ A. O. Caldeira and A. J. Leggett, Ann. Phys. 149, 374 (1983).

${ }^{76}$ A. J. Leggett, S. Chakravarty, A. T. Dorsey, M. P. A. Fisher, A. Garg, and W. Zwerger, Rev. Mod. Phys. 59, 1 (1987).

${ }^{77}$ M. Topaler and N. Makri, J. Chem. Phys. 101, 7500 (1994).

${ }^{78}$ L. Verlet, Phys. Rev. 159, 98 (1967).

${ }^{79}$ S. Habershon, D. E. Manolopoulos, T. E. Markland, and T. F. Miller III, "Ring-polymer molecular dynamics: Quantum effects in chemical dynamics from classical trajectories in an extended phase space," Annu. Rev. Phys. Chem. (published online).

${ }^{80}$ S. Kumar, D. Bouzida, R. H. Swendsen, P. A. Kollman, and J. M. Rosenberg, J. Comput. Chem. 13, 1011 (1992).

${ }^{81}$ S. Kumar, J. M. Rosenberg, D. Bouzida, R. H. Swendsen, and P. A. Kollman, J. Comput. Chem. 16, 1339 (1995).

${ }^{82}$ H. C. Andersen, J. Comput. Phys. 52, 24 (1983).
${ }^{83}$ G. Hummer, J. Chem. Phys. 120, 516 (2004).

${ }^{84}$ E. W. Vanden-Eijnden, J. Stat. Phys. 123, 503 (2006).

${ }^{85}$ A. Warshel, J. Phys. Chem. 86, 2218 (1982).

${ }^{86}$ G. King and A. Warshel, J. Chem. Phys. 93, 8682 (1990).

${ }^{87}$ D. W. Small, D. V. Matyushov, and G. A. Voth, J. Am. Chem. Soc. 125, 7470 (2003).

${ }^{88}$ D. T. Colbert and W. H. Miller, J. Chem. Phys. 96, 1982 (1992).

${ }^{89}$ N. Ananth and T. F. Miller III, Mol. Phys. 110, 1009 (2012).

${ }^{90}$ J. O. Richardson and S. C. Althorpe, J. Chem. Phys. 131, 214106 (2009).

${ }^{91}$ S. C. Althorpe, J. Chem. Phys. 134, 114104 (2011).

${ }^{92}$ A. Kuki and P. G. Wolynes, Science 236, 1647 (1987).

${ }^{93}$ D. M. Ceperley, Rev. Mod. Phys. 67, 279 (1995).

${ }^{94}$ M. Marchi and D. Chandler, J. Chem. Phys. 95, 889 (1991).

${ }^{95}$ J. O. Richardson and S. C. Althorpe, J. Chem. Phys. 134, 054109 (2011).

${ }^{96}$ J. O. Richardson, S. C. Althorpe, and D. J. Wales, J. Chem. Phys. 135, 124109 (2011).

${ }^{97}$ D. Chandler, in Liquids, Freezing and the Glass Transition, Les Houches 51, edited by D. Levesque, J. P. Hansens, and J. Zinn-Justin (Elsevier Science Publishers, Amsterdam, 1991), pp. 193-285.

${ }^{98}$ S. Hammes-Schiffer, Chem. Phys. Chem. 3, 33 (2002).

${ }^{99}$ J. H. Skone, A. V. Soudackov, and S. Hammes-Schiffer, J. Am. Chem. Soc. 128, 16655 (2006).

${ }^{100}$ R. I. Cukier, Biochim. Biophys. Acta Bioenerg. 1655, 37 (2004).

${ }^{101}$ J. J. Warren, A. R. Menzeleev, J. S. Kretchmer, T. F. Miller III, H. B. Gray, and J. M. Mayer, J. Phys. Chem. Lett. 4, 519 (2013).

${ }^{102}$ H. B. Gray and J. R. Winkler, Proc. Natl. Acad. Sci. U.S.A. 102, 3534 (2005).

${ }^{103}$ M. Bixon and J. Jortner, J. Chem. Phys. 107, 5154 (1997).

${ }^{104}$ M. D. Newton, Chem. Rev. 91, 767 (1991).

${ }^{105}$ M. Cho, S. J. Rosenthal, N. F. Scherer, L. D. Ziegler, and G. R. Fleming, J. Chem. Phys. 96, 5033 (1992). 